\title{
Quasi-ordinary singularities, essential divisors and Poincaré series
}

\author{
P. D. González Pérez and F. Hernando
}

\begin{abstract}
We define Poincaré series associated to a germ $(S, 0)$ of toric or analytically irreducible quasiordinary hypersurface singularity, by a finite sequence of monomial valuations such that at least one of them is centered at the point 0 . This involves the definition of a multi-graded ring associated to the analytic algebra of the singularity by the sequence of valuations. We prove that the Poincare series is a rational function with integer coefficients, which can also be defined as an integral with respect to the Euler characteristic of a function defined by the valuations, over the projectivization of the analytic algebra of the singularity. In particular, the Poincaré series associated to the set of divisorial valuations of the essential divisors, considered both over the singular locus and over the point 0 , is an analytic invariant of the singularity. In the quasi-ordinary hypersurface case we prove that this Poincaré series determines and is determined by the normalized sequence of characteristic monomials. These monomials in the analytic case define a complete invariant of the embedded topological type of the hypersurface singularity.
\end{abstract}

\section{Introduction}

After the publication of Gusein-Zade, Delgado and Campillo's paper [19] there have been some positive results in the determination of topological invariants of complex analytic singularities by using Poincaré series associated to divisorial valuations. For instance, if $(S, 0)$ is a germ of plane curve and the valuations are those defined by the parametrizations of its irreducible components the Poincaré series is a rational function that encodes topological information of the singularity, namely its Alexander polynomial [5]. If $(S, 0)$ is a germ of rational surface singularity and the valuations are those associated with the irreducible components of the exceptional divisor of its minimal resolution the Poincaré series has a rational form with denominator determined by the intersection matrix of the exceptional divisor in the minimal resolution $[\mathbf{6}]$. See also $[\mathbf{8}-\mathbf{1 0}, \mathbf{3 1}]$.

The divisors corresponding to the irreducible components of the minimal embedded resolution of a germ of surface singularity provide the basic example of essential divisors. Roughly speaking, essential is a property of the associated divisorial valuation that must hold in every resolution of singularities. This notion is much more interesting if the dimension of the variety is at least 3 , since we do not dispose then of a minimal resolution of singularities. We say that the divisorial valuation associated to an essential divisor is also essential. Essential divisors appear in Nash's approach to studying singularities through the properties of arc spaces. Nash introduced a map from the set of irreducible components of the space of arcs with origin at the singular locus of a variety to the set of essential divisors over the singular locus. This map is called the Nash map. We can also consider a similarly defined map from the set of irreducible components of the space of arcs with origin at a fixed singular point and the divisors over this point which are essential. This map is called the local Nash map. Both maps are injective 
$($ see $[\mathbf{2 2}, \mathbf{3 0}])$ but there are examples of four-dimensional isolated hypersurface singularities for which the Nash map is not surjective (see $[\mathbf{2 2}]$ ).

With this view-point it is very natural to consider an invariant of the germ $(S, 0)$ defined by the Poincaré series associated to $(S, 0)$ by the finite sequence of essential divisorial valuations. We develop this program, providing a new intrinsic definition of the Poincaré series, when $(S, 0)$ is a toric singularity or an irreducible germ of quasi-ordinary hypersurface singularity, two classes of singularities that are not necessarily normal and not isolated in general.

Quasi-ordinary singularities arise classically in Jung's approach to analyze surface singularities by using a finite projection to a smooth surface (see $[\mathbf{2 3}]$ ). We say that a germ $(S, 0) \subset\left(\mathbf{C}^{d+1}, 0\right)$ of complex analytic hypersurface with equation $f=0$ is quasi-ordinary if there exists a finite morphism $\pi:(S, 0) \rightarrow\left(\mathbf{C}^{d}, 0\right)$ (called a quasi-ordinary projection) such that the discriminant locus is a germ of normal crossing divisor. Quasi-ordinary hypersurface singularities are parametrized by quasi-ordinary branches, a class of fractional power series in several variables having a finite set of distinguished or characteristic monomials, which generalize the characteristic pairs associated to a plane branch (see $[\mathbf{1}, \mathbf{2 6}]$ ). Lipman and Gau's results imply that the characteristic monomials of an analytically irreducible quasiordinary hypersurface determine a complete invariant of the embedded topological type (see $[\mathbf{1 3}, \mathbf{2 6}])$. Quasi-ordinary singularities also play an important role in the comprehension of the different notions of equisingularity, in particular with respect to Zariski equisingularity and equiresolution (see $[\mathbf{2}, \mathbf{2 7}]$ ).

Let $\underline{\nu}=\left(\nu_{1}, \ldots, \nu_{r}\right)$ be a sequence of monomial valuations on an irreducible germ $(S, 0)$ of toric or quasi-ordinary hypersurface singularity, such that at least one of them is centered at the point 0 . We prove that monomial valuations are compatible, a property which allows us to define a multi-graded ring, $\operatorname{gr}_{\underline{\underline{\nu}}} \mathcal{O}_{S}=\bigoplus_{\underline{a} \in \mathbf{Z}_{\geqslant 0}^{r}} J(\underline{a})$, associated to the analytic algebra $\mathcal{O}_{S}$. The homogeneous components $J(\underline{a})$ of this multi-graded ring are vector spaces of finite dimension over $\mathbf{C}$ (see Section 3$)$. We then define the Poincaré series of $(S, 0)$ relative to the sequence $\underline{\nu}$ as

$$
P_{(S, 0)}^{\underline{\nu}}:=\sum_{\underline{a} \in \mathbf{Z}_{\geqslant 0}^{r}} \operatorname{dim}_{\mathbf{C}}(J(\underline{a})) \underline{t}^{\underline{a}} \in \mathbf{Z}[[\underline{t}]],
$$

for $\underline{t}=\left(t_{1}, \ldots, t_{r}\right)$. This can be expressed as the Poincaré series of an extended semigroup associated with the multi-graded ring $\operatorname{gr}_{\underline{\nu}} \mathcal{O}_{S}$. The Poincaré series is a rational function in $\underline{t}$ with integer coefficients. We prove that the Poincaré series is equal to an integral with respect to the Euler characteristic, of a function defined by $\underline{\nu}$, on the projectivization of the analytic algebra of the singularity (see Theorems 4.2 and 4.4 ). In the quasi-ordinary case we prove that the Poincaré series can be expanded in a unique way as a product of terms of the form $\left(1-\underline{t}^{\underline{a}}\right)^{ \pm 1}$. Some of the methods introduced here might be of application to other classes of non-isolated singularities.

Notice that we cannot use Campillo, Delgado and Gusein-Zade's multi-index filtrations to define the Poincaré series if there is some monomial valuation in the sequence $\underline{\nu}$ which is not centered at the origin. In the toric case our definition and results generalize those obtained by using multi-index filtrations in a recent paper of Lemahieu [25].

The classes of singularities we are dealing with, toric and hypersurface quasi-ordinary, possess essential divisors that are invariant by the torus action of the normalization. These divisors then define essential divisorial valuations, which are monomial valuations. This characterization in different cases is done in $[\mathbf{3}, \mathbf{1 6}, \mathbf{2 0 - 2 2}]$. Since in our construction we need at least one valuation centered at the origin, we combine two different notions of essential valuation: those centered at the origin and those centered at some component of the singular locus of $S$.

A detailed combinatorial description of the essential divisors in the quasi-ordinary case in terms of the structure of the singular locus of $(S, 0)$ is given in Section 5 . Of essential use is Lipman's characterization of the singular locus of a quasi-ordinary hypersurface (see 
Theorem 2.1). The information on the normalized characteristic monomials provided by the essential valuations is encoded in a matrix $\mathcal{M}$, which is defined and studied in Subsection 5.1.

If $\underline{\nu}$ is the sequence of essential divisorial valuations, then the associated Poincaré series, $P_{(S, 0)}$, is an analytical invariant of the singularity. The main result of this paper is the following theorem (see Theorem 6.1).

Theorem. The Poincaré series $P_{(S, 0)}$ determines and is determined by the embedded topological type of $(S, 0) \subset\left(\mathbf{C}^{d+1}, 0\right)$.

The proof is constructive and passes through the analysis of the rational form of the Poincaré series, determining from it the dimension, the number of codimension-one components of the singular locus, the equisingular dimension (see Section 2) and finally the characteristic monomials by resolving a sequence of linear systems defined by certain minors of the matrix $\mathcal{M}$. The proof is divided into several cases depending on the dimension and the number of essential divisors over codimension-two irreducible components of the singular locus.

We find out that the Poincaré series characterizes topological equisingularity in an equisingular family of quasi-ordinary hypersurfaces (see Corollary 6.3).

In Subsection 6.1 we compare the zeta function of geometric monodromy with the Poincaré series in the quasi-ordinary hypersurface case. We find out that the zeta function appears as a factor of the rational function defined from the Poincare series by specializing the indeterminates $t_{1}, \ldots, t_{p}$ in one variable $t$. Corollary 6.5 gives a partial answer to McEwan and Némethi's question $\mathrm{H}$ in [28] and involves a new related question. We conclude the article with an example.

\section{A reminder of toric geometry}

We give some definitions and notation (see [11], [12] or [32] for proofs). If $N$ is a lattice, we denote by $M$ the dual lattice, by $N_{\mathbf{R}}$ the real vector space spanned by $N$ and by $\langle$, the canonical pairing between the dual lattices $N$ and $M$ (respectively vector spaces $N_{\mathbf{R}}$ and $M_{\mathbf{R}}$ ). A rational convex polyhedral cone $\tau$ in $N_{\mathbf{R}}$, a cone in what follows, is the set $\tau:=$ $\operatorname{pos}\left(a_{1}, \ldots, a_{s}\right)$ of non-negative linear combinations of vectors $a_{1}, \ldots, a_{s} \in N$. The cone $\tau$ is strictly convex if $\tau$ contains no linear subspace of dimension greater than 0 . We denote by $\stackrel{\circ}{\tau}$ the relative interior of a cone $\tau$. The dual cone $\tau^{\vee}$ (respectively orthogonal cone $\tau^{\perp}$ ) of $\tau$ is the set $\left\{w \in M_{\mathbf{R}} /\langle w, u\rangle \geqslant 0\right\}$ (respectively $\{\langle w, u\rangle=0 \forall u \in \tau\}$ ).

Let $\Lambda$ be a sub-semigroup of finite type of a rank $d$ lattice $M:=\Lambda+(-\Lambda)$ which it generates as a group. Any affine toric variety is of the form $Z^{\Lambda}=\operatorname{Spec} \mathbf{C}[\Lambda]$, where $\mathbf{C}[\Lambda]=$ $\left\{\sum_{\text {finite }} c_{\lambda} X^{\lambda} \mid c_{\lambda} \in \mathbf{C}\right\}$ is the $\mathbf{C}$-algebra of the semigroup. Fixing a basis of the lattice $M$ induces an embedding of $\Lambda$ in $\mathbf{Z}^{d}$, corresponding to an inclusion of $\mathbf{C}[\Lambda]$ in the ring of Laurent polynomials $\mathbf{C}\left[y_{1}^{ \pm 1}, \ldots, y_{d}^{ \pm 1}\right], X^{e} \mapsto y_{1}^{e_{1}} \cdots y_{d}^{e_{d}}$, for $\left(e_{1}, \ldots, e_{d}\right)$ the coordinates of the vector $e \in \Lambda$ with respect to the basis of the lattice. The torus $Z^{M}$ is an open dense subset of $Z^{\Lambda}$, which acts on $Z^{\Lambda}$, and the action extends the action of the torus on itself by multiplication. The semigroup $\Lambda$ spans the cone $\sigma^{\vee}=\mathbf{R}_{\geqslant 0} \Lambda$, and thus we have an inclusion of semigroups $\Lambda \rightarrow \bar{\Lambda}:=\sigma^{\vee} \cap M$, defining an associated toric modification $Z_{\sigma}:=Z^{\bar{\Lambda}} \rightarrow Z^{\Lambda}$, which is the normalization map. The cone $\sigma^{\vee}$ has a vertex if and only if there exists a zerodimensional orbit, and in this case this orbit is reduced to the origin $0 \in Z^{\Lambda}$ defined by the maximal ideal $\mathfrak{m}_{\Lambda}:=\left(X^{\lambda} \mid \lambda \in \Lambda-\{0\}\right) \mathbf{C}[\Lambda]$. The ring $\mathbf{C}\{\Lambda\}$ denotes the local ring of germs of holomorphic functions at $\left(Z^{\Lambda}, 0\right)$. We denote by $\mathbf{C}[[\Lambda]]$ the completion of $\mathbf{C}\{\Lambda\}$ with respect to its maximal ideal $\mathfrak{m}_{\Lambda} \mathbf{C}\{\Lambda\}$. Any non-zero vector $v \in \sigma \cap N$ defines a valuation $\nu$ of the field of fractions of $\mathbf{C}\{\Lambda\}$, called monomial valuation or toric valuation, which is defined for an 
element $0 \neq \phi=\sum c_{u} X^{u} \in \mathbf{C}\{\Lambda\}$ by $\nu(\phi)=\min _{c_{u} \neq 0}\langle v, u\rangle$. If the vector $v$ is primitive, then the valuation $\nu$ is divisorial (see $[\mathbf{3}],[\mathbf{4}]$ or $[\mathbf{1 7}]$ ).

\section{Quasi-ordinary singularities}

A germ of complex analytic hypersurface $(S, 0) \subset\left(\mathbf{C}^{d+1}, 0\right)$ is quasi-ordinary if there exists a finite morphism $(S, 0) \rightarrow\left(\mathbf{C}^{d}, 0\right)$ (called a quasi-ordinary projection) such that the discriminant locus is contained (germ-wise) in a normal crossing divisor. In suitable coordinates depending on this projection, the hypersurface $(S, 0)$ has an equation $f=0$, where $f \in \mathbf{C}\{\underline{x}\}[y]$ is a quasiordinary polynomial: a Weierstrass polynomial such that the discriminant $\Delta_{y} f$, as a polynomial in $y$, is of the form $\Delta_{y} f=\underline{x}^{\delta} \epsilon$, where $\epsilon$ is a unit in the ring $\mathbf{C}\{\underline{x}\}$ of convergent power series in the variables $\underline{x}=\left(x_{1}, \ldots, x_{d}\right)$ and $\delta \in \mathbf{Z}_{\geqslant 0}^{d}$. We assume from now on that the germ $(S, 0)$ is irreducible, that is, the polynomial $f$ is irreducible.

In this section we recall some numerical data that are associated to the fixed quasi-ordinary projection or, equivalently, to the quasi-ordinary polynomial $f$. We use these data to describe the singular locus and some invariants of the germ $(S, 0)$.

The Jung-Abhyankar theorem guarantees that all the roots $\left\{\zeta^{(l)}\right\}_{l=1}^{n}$ of $f$, which are called quasi-ordinary branches, are fractional power series in the ring $\mathbf{C}\left\{\underline{x}^{1 / n}\right\}$ for $\underline{x}^{1 / n}=$ $\left(x_{1}^{1 / n}, \ldots, x_{d}^{1 / n}\right)$ and $n:=\operatorname{deg} f \in \mathbf{Z}_{\geqslant 0}$ (see [1] ). Since the discriminant of $f$ is equal to $\Delta_{y} f=$ $\prod_{i \neq j}\left(\zeta^{(i)}-\zeta^{(j)}\right)$, each factor $\zeta^{(i)}-\zeta^{(j)}$ is of the form a monomial times a unit in $\mathbf{C}\left\{\underline{x}^{1 / n}\right\}$. These monomials (respectively their exponents) are called characteristic or distinguished, of the quasi-ordinary polynomial $f$. These monomials depend on the choice of quasi-ordinary projection. The characteristic exponents can be relabeled in the form

$$
\lambda_{1}<\lambda_{2}<\ldots<\lambda_{g},
$$

where $<$ means $\leqslant$ coordinate-wise and $\neq($ see $[\mathbf{2 6}])$. Without loss of generality we relabel the variables $x_{1}, \ldots, x_{d}$ in such a way that if $\lambda_{j}=\left(\lambda_{j}^{1}, \ldots, \lambda_{j}^{d}\right) \in \mathbf{Q}^{d}$ for $j=1, \ldots, g$, then we have

$$
\left(\lambda_{1}^{1}, \ldots, \lambda_{g}^{1}\right) \geqslant_{\text {lex }} \ldots \geqslant_{\text {lex }}\left(\lambda_{1}^{d}, \ldots, \lambda_{g}^{d}\right),
$$

where $\geqslant_{\text {lex }}$ is lexicographic order.

Definition 1. The characteristic exponents $\lambda_{1}, \ldots, \lambda_{g}$ of a quasi-ordinary polynomial $f$ are normalized if (2.2) is verified and, in addition, if $\lambda_{1}=\left(\lambda_{1}^{1}, 0, \ldots, 0\right)$ then $\lambda_{1}^{1}>1$.

If this is the case, we say that the quasi-ordinary polynomial $f$ or the associated quasiordinary projections are also normalized. Lipman showed how to associate a normalized quasiordinary polynomial $f^{\prime}$ to a given quasi-ordinary polynomial $f$ in such a way that $f=0$ and $f^{\prime}=0$ define the same germ and the characteristic exponents associated to $f^{\prime}$ are related with those associated to $f$ by certain inversion formulas (see Lipman's Appendix in [13]).

REMARK 1. We assume that the characteristic exponents and quasi-ordinary polynomials are normalized unless otherwise stated.

Recall that two hypersurface germs $\left(S_{i}, 0\right) \subset\left(\mathbf{C}^{d+1}, 0\right)$ for $i=1,2$ have the same embedded topological type if there exists a germ homeomorphism $\left(\mathbf{C}^{d+1}, S_{1}, 0\right) \rightarrow\left(\mathbf{C}^{d+1}, S_{2}, 0\right)$ of triples. Two irreducible germs of $d$-dimensional quasi-ordinary hypersurface $\left(S_{i}, 0\right) \subset\left(\mathbf{C}^{d+1}, 0\right)$ for $i=$ 1,2 have the same embedded topological type if and only if both have the same sequence of normalized characteristic monomials (see $[\mathbf{1 3}, \mathbf{2 6}]$ ). 
Definition 2. The characteristic exponents determine the following nested sequence of characteristic lattices: $M_{0}:=\mathbf{Z}^{d} \subset M_{1} \subset \ldots \subset M_{g}=: M$, where $M_{j}:=M_{j-1}+\mathbf{Z} \lambda_{j}$ for $j=1, \ldots, g$ with the convention $\lambda_{g+1}=+\infty$. The index $n_{j}$ of the lattice $M_{j-1}$ in the lattice $M_{j}$ is greater than 1 . We call $n_{1}, \ldots, n_{g}$ the characteristic integers associated to $f$ (see $[\mathbf{1 4}, \mathbf{2 6}, \mathbf{3 4}]$ ).

Suppose that the reduced discriminant locus of the quasi-ordinary projection $\pi$ has equation $x_{1} \cdots x_{c}=0$. The integer $c$ is called in [34] the equisingular dimension of $(S, 0)$, since $(S, 0)$ may be viewed as a topologically equisingular deformation of a quasi-ordinary hypersurface germ of dimension $c$, but not smaller. See [2] and also Lipman's work [27]. The following result is a reformulation of part of [26, Theorem 7.3], using our notation.

TheOREm 2.1. With the previous notation, we have that the singular locus Sing $S$ of the quasi-ordinary hypersurface $(S, 0)$ has only irreducible components of codimension one or two of the form $Z_{i}:=S \cap\left\{x_{i}=0\right\}$ or $Z_{i, j}:=S \cap\left\{x_{i}=x_{j}=0\right\}$, for $1 \leqslant i, j \leqslant c$. If $1 \leqslant i \leqslant c$, the coordinate section $Z_{i}$ is a component unless $\lambda_{1}^{i}=\ldots=\lambda_{g-1}^{i}=0$ and $\lambda_{g}^{i}=1 / n_{g}$. If $1 \leqslant i, j \leqslant c$, the coordinate section $Z_{i, j}$ is a component if and only if neither $Z_{i}$ nor $Z_{j}$ is a component of the singular locus of $S$.

Since the polynomial $f$ is irreducible, we can identify the analytic algebra $\mathcal{O}_{S}:=\mathbf{C}\{\underline{x}\}[y] /(f)$ of the germ $(S, 0)$ with the ring $\mathbf{C}\{\underline{x}\}[\zeta]$ for $\zeta$ any fixed quasi-ordinary branch $\zeta$ parametrizing $(S, 0)$.

We denote by $N$ (respectively by $N_{j}$ ) the dual lattice of $M$ (respectively of $M_{j}$, for $j=$ $1, \ldots, g)$ and by $\sigma \subset N_{\mathbf{R}}$ the cone spanned by the dual basis of the canonical basis of $M_{0}$. With this notation we have that the homomorphism $\mathbf{C}\{\underline{x}\} \rightarrow \mathbf{C}\left\{\sigma^{\vee} \cap M_{0}\right\}$ which maps $x_{i} \mapsto X^{e_{i}}$, for $e_{i}$ running through the primitive integral vectors of the lattice $M_{0}$ in the edges of $\sigma^{\vee}$, is an isomorphism of $\mathbf{C}$-algebras since $\left\{e_{1}, \ldots, e_{d}\right\}$ is a basis of $M_{0}$. Note that $X^{e}$ denotes a monomial of the semigroup algebra, without making any reference to a choice of basis of the lattice. Since the exponents of the quasi-ordinary branch $\zeta$ belong to the semigroup $\sigma^{\vee} \cap M$, we obtain a ring extension $\mathcal{O}_{S}=\mathbf{C}\left\{\sigma^{\vee} \cap M_{0}\right\}[\zeta] \rightarrow \mathbf{C}\left\{\sigma^{\vee} \cap M\right\}$, which is the inclusion of $\mathcal{O}_{S}$ in its integral closure in its field of fractions.

Proposition 2.2 ([15, Proposition 14]). The map $n:\left(Z_{\sigma}, 0\right) \rightarrow(S, 0)$ corresponding to the ring extension

$$
\mathbf{C}\left\{\sigma^{\vee} \cap M_{0}\right\}[\zeta] \longrightarrow \mathbf{C}\left\{\sigma^{\vee} \cap M\right\}
$$

is the normalization map of $(S, 0)$.

More generally, the normalization of a germ of quasi-ordinary singularity, not necessarily hypersurface, is a toric singularity (see [34]).

The elements of $M$ defined by

$$
\gamma_{1}=\lambda_{1} \quad \text { and } \quad \gamma_{j+1}-n_{j} \gamma_{j}=\lambda_{j+1}-\lambda_{j} \quad \text { for } j=1, \ldots, g-1
$$

together with the semigroup $\Gamma_{\pi}:=\mathbf{Z}_{\geqslant 0}^{d}+\gamma_{1} \mathbf{Z}_{\geqslant 0}+\ldots+\gamma_{g} \mathbf{Z}_{\geqslant 0} \subset \sigma^{\vee} \cap M_{\mathbf{Q}}$ are associated with the quasi-ordinary projection $\pi$.

REMARK 2. For any $\gamma \in \Gamma_{\pi}$ there exists a function $\phi_{\gamma} \in \mathcal{O}_{S}$ such that $\phi_{\gamma}=X^{\gamma} \cdot \epsilon_{\gamma}$ in $\mathbf{C}\left\{\sigma^{\vee} \cap M\right\}$, where $\epsilon_{\gamma}$ is a unit in $\mathbf{C}\left\{\sigma^{\vee} \cap M\right\}$ (see [14], [15] or [34]). 
We have that for $1 \leqslant j<g$

$$
n_{j} \gamma_{j}<\gamma_{j+1}
$$

where $<$ means $\neq$ and $\leqslant$ coordinate-wise. The equality

$$
\gamma=\alpha+l_{1} \gamma_{1}+\ldots+l_{g} \gamma_{g} \quad \text { with } \alpha \in \sigma^{\vee} \cap M_{0}, 0 \leqslant l_{i}<n_{i}, i=1, \ldots, g,
$$

determines an element $\gamma$ of the semigroup $\Gamma_{\pi}$ and any element of this semigroup has a unique expansion of the form (2.6) (see [14, Lemma 3.3]).

Definition 3. The generating series of the semigroup $\Gamma_{\pi}$ is $P_{\Gamma_{\pi}}:=\sum_{\gamma \in \Gamma_{\pi}} X^{\gamma} \in \mathbf{C}\left[\left[\Gamma_{\pi}\right]\right]$.

Proposition 2.3. The generating series of the semigroup $\Gamma_{\pi}$ is a rational function:

$$
P_{\Gamma_{\pi}}=\prod_{j=1}^{d} \frac{1}{1-X^{e_{j}}} \prod_{i=1}^{g} \frac{1-X^{n_{i} \gamma_{i}}}{1-X^{\gamma_{i}}} \in \mathbf{C}\left(\Gamma_{\pi}\right),
$$

where $\mathbf{C}\left(\Gamma_{\pi}\right)$ denotes the field of fractions of $\mathbf{C}\left[\Gamma_{\pi}\right]$.

Proof. We use the unique expansion form (2.6) of the elements of the semigroup

$$
P_{\Gamma_{\pi}}=\sum_{\gamma} X^{\alpha(\gamma)} X^{\sum l_{i}(\gamma) \gamma_{i}} \stackrel{(2.6)}{=} \sum_{\alpha \in \sigma^{\vee} \cap M_{0}} X^{\alpha} \prod_{i=1}^{g}\left(1+X^{\gamma_{i}}+\cdots+X^{\left(n_{i}-1\right) \gamma_{i}}\right) .
$$

Notice that by construction the semigroup, $\sigma^{\vee} \cap M_{0}$ is isomorphic to $\mathbf{Z}_{\geqslant 0}^{d}$. Then, rewriting this expression using elementary properties of the geometric series gives the result.

In principle, the semigroup $\Gamma_{\pi}$ depends on the choice of quasi-ordinary projection. In the embedded surface case Kiyek and Micus [24] proved the analytic invariance of the semigroup $\Gamma_{\pi}$ (see also $[33]$ ). In arbitrary dimension, Popescu-Pampu proved the invariance of the semigroup $\Gamma_{\pi}$ by analyzing the preimage of the singular locus of $(S, 0)$ by the orbifold mapping associated to $(S, 0)$ and certain plane sections transversal to the singular locus. Another proof of the analytical invariance of $\Gamma_{\pi}$ was given by the first author and Gonzalez-Sprinberg in $[\mathbf{1 7}]$, by considering the graded rings associated to the divisorial valuations of the irreducible components of the exceptional divisor of the normalized blow-up of $0 \in S$ (see also [14]).

\section{The graded ring associated with finitely many monomial valuations}

Lejeune noticed that the semigroup of a plane branch is determined by the graded ring associated to the filtration induced on its analytic algebra by the maximal ideal of its integral closure (see [37, p. 161]). We generalize this result by defining a graded ring associated to the analytic algebra of a toric or a quasi-ordinary singularity by a sequence of toric divisorial valuations. In this section we extend the approach and the results obtained in $[\mathbf{1 7}]$ (corresponding to the case of a single valuation).

A discrete valuation $\nu$ of the field of fractions of a ring $R$, which is centered on $R$, defines a filtration of $R$ with ideals $I(b)=\{\phi \in R \mid \nu(\phi) \geqslant b\}$ for $b \in \mathbf{Z}_{\geqslant 0}$. The associated graded ring is $\operatorname{gr}_{\nu} R:=\bigoplus_{b \geqslant 0} I(b) / I(b+1)$.

Suppose now that the ring $R$ is graded over the semigroup $\mathbf{Z}_{\geqslant 0}^{r}$; that is, it is of the form $R:=\bigoplus_{\underline{a} \in \mathbf{Z}_{\geqslant 0}^{r}} J(\underline{a})$, where $J(\underline{a})$ denotes the homogeneous component associated to $\underline{a} \in \mathbf{Z}_{\geqslant 0}^{r}$. In this case we say that $R$ is multi-graded or simply that $R$ is graded. In general, an ideal $I(b) \subset R$ associated to the valuation is not homogeneous with respect to the grading of $R$. 
Definition 4. We say that the valuation $\nu$ is compatible with the graduation if for all $b \geqslant 0$ the ideal $I(b)$ is a homogeneous ideal, that is,

$$
I(b)=\bigoplus_{\underline{a} \in \mathbf{Z}_{\geqslant 0}^{r}} I(\underline{a}, b), \quad \text { where } I(\underline{a}, b):=\{\phi \in J(\underline{a}) \mid \nu(\phi) \geqslant b\} .
$$

It follows that the graded ring $\operatorname{gr}_{\nu} R$ inherits a multi-grading since $I(b) / I(b+1)=$ $\bigoplus_{a \in \mathbf{Z}_{\geqslant 0}^{r}} I(\underline{a}, b) / I(\underline{a}, b+1)$. If we denote $J(\underline{a}, b):=I(\underline{a}, b) / I(\underline{a}, b+1)$, then we have that $\operatorname{gr}_{\nu} R:=\bigoplus_{\underline{a} \in \mathbf{Z}_{\geqslant 0}^{r}, b \geqslant 0} J(\underline{a}, b)$.

REMARK 3. This notion of compatibility of a valuation with a graded ring can be defined more generally for non-discrete valuations and for gradings over different semigroups, though we do not need this in this paper.

\subsection{Toric case}

Let $\left(Z^{\Lambda}, 0\right)$ be the germ of an affine toric variety at its zero orbit, which is assumed to exist. The normalization $Z^{\bar{\Lambda}}$ is of the form $Z^{\bar{\Lambda}}=Z_{\sigma, N}$.

Let $\underline{v}=\left(v_{1}, \ldots, v_{r}\right)$ be a finite sequence of non-zero vectors in $\sigma \cap N$. We consider the graduation of the $\mathbf{C}$-algebra $\mathbf{C}[\Lambda]$,

$$
\mathbf{C}[\Lambda]^{(\underline{v})}:=\bigoplus_{\underline{a} \in \mathbf{Z}_{\geqslant 0}^{r}} H(\underline{a}),
$$

where the homogeneous terms $H(\underline{a})$ are defined by

$$
H(\underline{a}):=\bigoplus_{\left\langle v_{j}, u\right\rangle=a_{j}, j=1, \ldots, r}^{u \in \Lambda} \mathbf{C} X^{u} \quad \text { for } \underline{a}=\left(a_{1}, \ldots, a_{r}\right) \in \mathbf{Z}_{\geqslant 0}^{r} .
$$

We show that the graduation $\mathbf{C}[\Lambda]^{(\underline{v})}$ introduced above is in fact a multi-grading associated to the sequence of monomial valuations $\nu_{1}, \ldots, \nu_{r}$ corresponding to the sequence of vectors $v_{1}, \ldots, v_{r} \in \sigma \cap N-\{0\}$. Let $v \in \sigma \cap N$ be a non-zero vector defining a monomial valuation $\nu$ of the ring $R$, equal to $\mathbf{C}\{\Lambda\}$ (or to $\mathbf{C}[\Lambda]$ ). The associated graded ring is $\operatorname{gr}_{\nu} R=\bigoplus_{a \geqslant 0} J(a)$, where $J(a)=I(a) / I(a+1)$ and $I(a)$ denotes the ideals of the filtration associated to $\nu$. If $0 \neq h \in \mathbf{C}\{\Lambda\}$ then $h_{\mid v}$ denotes the symbolic restriction of $h$ to the face defined by the vector $v$ on its Newton polyhedron (see $[\mathbf{1 7}]$ ).

Proposition 3.1. With the above notation, the mapping $J(a)-\{0\} \rightarrow H(a)-\{0\}$ given by $h \bmod I(a+1) \mapsto h_{\mid v}$, extends to an isomorphism of graded rings

$$
\psi: g r_{\nu} R \longrightarrow \mathbf{C}[\Lambda]^{(v)} .
$$

If $0 \neq w \in \sigma \cap N$ defines a monomial valuation $\omega$ of $R$, then define $\bar{\omega}: g r_{\nu} R-\{0\} \rightarrow \mathbf{Z}_{\geqslant 0}$ on homogeneous elements by

$$
J(a)-\{0\} \longrightarrow \mathbf{Z}_{\geqslant 0}, \quad \bar{h} \longmapsto \max \{\omega(h) \mid h+I(a+1)=\bar{h}\}
$$

and, in general, if $0 \neq \phi=\sum_{a} \bar{h}_{a} \in \operatorname{gr}_{\nu} R$ is a sum of homogeneous terms $0 \neq \bar{h}_{a} \in J(a)$, then define $\bar{\omega}(\phi):=\min _{a}\left\{\bar{\omega}\left(\bar{h}_{a}\right)\right\}$. Then we have that $\bar{\omega}=\omega \circ \psi$, and hence $\bar{\omega}$ is a monomial valuation of $\operatorname{gr}_{\nu} R$ compatible with the graded structure. 
Proof. For the first assertion see the proof of [17, Proposition 3.2]. For the second assertion we use the fact that $\psi$ is an isomorphism of graded rings, and hence induces an isomorphism of homogeneous components $J(a) \rightarrow H(a)$.

If $0 \neq \bar{h} \in J(a)$, let us take a representative $h_{0} \in I(a)-I(a+1)$. Then we have $\psi(\bar{h})=$ $\left(h_{0}\right)_{\mid v} \in H(a)$. Also notice that $\left(h_{0}\right)_{\mid v}+I(a+1)=\bar{h}$ and that $\max \left\{\omega\left(\left(h_{0}\right)_{\mid v}+h^{\prime}\right) \mid h^{\prime} \in I(a+\right.$ $1)\}$ is reached, for instance, when $h^{\prime}=0$. This implies that on each homogeneous component $J(a)$ we have that $\bar{\omega}=\omega \circ \psi$. Notice now that both maps $\omega \circ \psi$ and $\bar{\omega}$ coincide on $\operatorname{gr}_{\nu} R$. This implies that $\bar{\omega}$ is a monomial valuation of $\operatorname{gr}_{\nu} R$, with respect to the toric structure defined by $\psi$.

We call $\bar{\omega}$ an induced valuation on the graded $\operatorname{ring} \operatorname{gr}_{\nu} R$.

Proposition 3.2. Let $\underline{\nu}=\left(\nu_{1}, \ldots, \nu_{r}\right)$ be a sequence of monomial valuations of the ring $R$ equal to $\mathbf{C}[\Lambda]$ or $\mathbf{C}\{\Lambda\}$; then these valuations induce a multi-graded ring $\operatorname{gr}_{\nu} R$ given with a sequence of valuations $\underline{\bar{\nu}}=\left(\bar{\nu}_{1}, \ldots, \bar{\nu}_{r}\right)$ on it, and there exists an isomorphism of graded rings $\psi: \operatorname{gr}_{\underline{\nu}} R \rightarrow \mathbf{C}[\Lambda]^{(\underline{v})}$ such that $\nu_{i} \circ \psi=\bar{\nu}_{i}$ for $i=1, \ldots, r$.

Proof. We generalize the proof of Proposition 3.1 by induction on $r$. At the step $k$ we have by induction hypothesis a graded ring $\operatorname{gr}_{\left(\nu_{1}, \ldots, \nu_{k}\right)} R$ with valuations $\tilde{\nu}_{i}$ and a graded isomorphism $\tilde{\psi}: \operatorname{gr}_{\left(\nu_{1}, \ldots, \nu_{k}\right)} R \rightarrow \mathbf{C}[\Lambda]^{\left(v_{1}, \ldots, v_{k}\right)} \operatorname{such}$ that $\nu_{i} \circ \tilde{\psi}=\tilde{\nu}_{i}$ for $i=1, \ldots, k$. This implies that $\tilde{\nu}_{i}$ can be seen as a monomial valuation of $\operatorname{gr}_{\left(\nu_{1}, \ldots, \nu_{k}\right)} R$, which is compatible with the graded structure. If $k<r$, it follows from Proposition 3.1 that $\tilde{\psi}$ induces a graded isomorphism $\bar{\psi}: \operatorname{gr}_{\left(\nu_{1}, \ldots, \nu_{k+1}\right)} R \rightarrow \mathbf{C}[\Lambda]^{\left(v_{1}, \ldots, v_{k+1}\right)}$, which is given in homogeneous components with the above notation by

$$
\begin{aligned}
J\left(a_{1}, \ldots, a_{k+1}\right)-\{0\} & \longrightarrow H\left(a_{1}, \ldots, a_{k+1}\right)-\{0\}, \\
h \bmod I_{\nu_{k+1}}\left(a_{1}, \ldots, a_{k}, a_{k+1}+1\right) & \longmapsto(\tilde{\psi}(h))_{\mid v_{k+1}} .
\end{aligned}
$$

Notice that this is a natural way of extending the map $\psi$ defined in Proposition 3.1 and makes $\bar{\psi}$ an isomorphism of the graded rings quoted above. Then the last assertion on the induced valuations is implied by Proposition 3.1.

REMARK 4. Taking a different order on the monomial valuations in Proposition 3.2 provides isomorphic pairs of graded rings with induced valuations. The graded ring $\operatorname{gr}_{\nu}(R)$ is graded on the semigroup $\langle\underline{v}, \Lambda\rangle=\left\{\langle\underline{v}, \gamma\rangle:=\left(\left\langle\underline{v}_{1}, \gamma\right\rangle, \ldots,\left\langle\underline{v}_{r}, \gamma\right\rangle\right) \mid\right.$ for $\left.\gamma \in \Lambda\right\} \subset \mathbf{Z}_{\geqslant 0}^{r}$. Indeed, we have that $\underline{a} \in\langle\underline{v}, \Lambda\rangle$ if and only if $J(\underline{a}) \neq(0)$.

\subsection{Quasi-ordinary hypersurface case}

Let $(S, 0)$ be an irreducible germ of quasi-ordinary hypersurface given with a normalized quasiordinary projection $\pi$, inducing a toric structure on the normalization $(\bar{S}, 0)=\left(Z_{\sigma, N}, o_{\sigma}\right)$. Any vector $v \in \sigma \cap N$ defines a monomial valuation on $\mathbf{C}\left\{\sigma^{\vee} \cap M\right\}$, and hence on $\mathcal{O}_{S} \subset \mathbf{C}\left\{\sigma^{\vee} \cap\right.$ $M\}$ by the inclusion (2.3). The associated graded ring is $\operatorname{gr}_{\nu} \mathcal{O}_{S}=\bigoplus_{a \geqslant 0} J(a)$, where $J(a)=$ $I(a) / I(a+1)$ and $I(a)$ denotes the ideals of the filtration associated to $\nu$.

Proposition 3.3. Let $\underline{\nu}=\left(\nu_{1}, \ldots, \nu_{r}\right)$ be a sequence of monomial valuations of the ring $R=\mathcal{O}_{S}$, with at least one, say $\nu_{1}$, centered at the maximal ideal of $R$. This sequence of valuations induces a multi-graded ring gr $\underline{\underline{\nu}} R$ given with a sequence of valuations $\underline{\underline{\nu}}=\left(\bar{\nu}_{1}, \ldots, \bar{\nu}_{r}\right)$ 
on it, and there exists an isomorphism of graded rings $\psi: \operatorname{gr}_{\nu} R \rightarrow \mathbf{C}\left[\Gamma_{\pi}\right]^{(\underline{v})}$ such that $\nu_{i} \circ \psi=\bar{\nu}_{i}$ for $i=1, \ldots, r$.

Proof. The sequence $\underline{\nu}$ corresponds to a sequence of vectors $\underline{v}=\left(v_{1}, \ldots, v_{r}\right)$ in $\sigma \cap N$, with $v_{1} \in \stackrel{\circ}{\sigma} \cap N$. The assertion corresponding to the case of one monomial valuation, necessarily centered at the maximal ideal of $R$ is similar to the statement of Proposition 3.1. For the proof see [17, Proposition 3.2]. Note that the hypothesis, $v_{1} \in \stackrel{\circ}{\sigma} \cap N$, is essential in this case. The rest of the assertion follows formally from the proof of Proposition 3.2.

The graded ring $\operatorname{gr}_{\underline{\nu}}\left(\mathcal{O}_{S}\right)$ is graded on the semigroup $\left\langle\underline{v}, \Gamma_{\pi}\right\rangle:=\left\{\langle\underline{v}, \gamma\rangle:=\left(\left\langle\underline{v}_{1}, \gamma\right\rangle, \ldots,\left\langle\underline{v}_{r}, \gamma\right\rangle\right) \mid\right.$ for $\left.\gamma \in \Gamma_{\pi}\right\} \subset \mathbf{Z}_{\geqslant 0}^{r}$.

REMARK 5. If $\tau$ is a permutation of $\{1, \ldots, r\}$ such that $v_{\tau(1)} \in \stackrel{\circ}{\sigma} \cap N$, then the sequences of vectors $\underline{v}$ and $\underline{v}_{\tau}:=\left(v_{\tau(1)}, \ldots, v_{\tau(r)}\right)$ define isomorphic pairs of graded rings with induced valuations.

\section{Poincaré series and integration}

Given a finite sequence $\underline{\nu}=\left(\nu_{1}, \ldots, \nu_{r}\right)$ of divisorial valuations, Campillo, Delgado and GuseinZade introduced the Poincaré series by defining a multi-index filtration by ideals of the analytic algebra of the singularity. The computation of the Poincare series passes through the integration with respect to the Euler characteristic of a function defined by the valuations, on the projectivization of the algebra of the singularity (see [5-7]).

We define a Poincaré series associated to a toric singularity $\left(Z^{\Lambda}, 0\right)$ (respectively a quasiordinary singularity $(S, 0))$ by a finite sequence $\underline{\nu}=\left(\nu_{1}, \ldots, \nu_{r}\right)$ of monomial valuations with at least one, say $\nu_{1}$, centered at the origin. The definition passes through the extended semigroup associated with the graded ring $\operatorname{gr}_{\nu} R$ for $R=\mathbf{C}\{\Lambda\}$ (respectively $R=\mathcal{O}_{S}$ ). We then prove that this Poincaré series is an integral with respect to the Euler characteristic of a certain function defined by $\underline{\nu}$, over the projectivization of $R$.

\subsection{Toric case}

Following Campillo, Delgado and Gusein-Zade, we consider a notion of an extended semigroup, $\widetilde{\Lambda}:=\{(\underline{a}, \phi) \mid \underline{a} \in\langle\underline{v}, \Lambda\rangle$ and $\phi \in J(\underline{a})\}$, associated to the graded ring $\operatorname{gr}_{\underline{\nu}} R=$ $\bigoplus_{\underline{a} \in\langle\underline{v}, \Lambda\rangle} J(\underline{a})$, for $R=\mathbf{C}\{\Lambda\}$ or $\mathbf{C}[\Lambda]$. The operation in the semigroup $\widetilde{\Lambda}$ is additive in the first entry and multiplicative in the second. The projection $\rho: \widetilde{\Lambda} \rightarrow \mathbf{Z}^{r}$ has fibers $\rho^{-1}(\underline{a})=J(\underline{a})$ if $\underline{a} \in\langle\underline{v}, \Lambda\rangle$ and $\rho^{-1}(\underline{a})=\{0\}$ otherwise.

If $L$ is a $\mathbf{C}$-vector space, we denote by $\mathbf{P} L$ its projectivization. Notice that if the dimension of $L$ is finite, then it is equal to the Euler characteristic $\chi(\mathbf{P} L)$.

If the sequence of monomial valuations $\underline{\nu}=\left(\nu_{1}, \ldots, \nu_{r}\right)$ corresponds to the sequence of vectors $\underline{v}=\left(v_{1}, \ldots, v_{r}\right)$, then our hypothesis that $\nu_{1}$ is centered at the origin is equivalent to $v_{1} \in \stackrel{\circ}{\sigma} \cap N$. This implies that for any $\underline{a}$ we have

$$
\operatorname{dim}_{\mathbf{C}} J(\underline{a})=\chi(\mathbf{P} J(\underline{a}))<\infty .
$$

Definition 5. The Poincaré series associated to the germ $\left(Z^{\Lambda}, 0\right)$ and the sequence of monomial valuations $\underline{\nu}$ is

$$
P_{\left(Z^{\Lambda}, 0\right)}^{\nu}:=\sum_{\underline{a} \in \mathbf{Z}_{\geqslant 0}^{r}} \chi(\mathbf{P} J(\underline{a})) \underline{t}^{\underline{a}} \in \mathbf{Z}[[\underline{t}]]
$$


REmark 6. We often denote $P_{\left(Z^{\Lambda}, 0\right)}^{\underline{\nu}}$ by $P_{\left(Z^{\Lambda}, 0\right)}^{\underline{v}}$, for $\underline{v}$ the sequence of vectors in $\sigma \cap N$ defining the sequence of monomial valuations $\underline{\nu}$.

TheOREm 4.1. Let $\underline{v}=\left(v_{1}, \ldots, v_{r}\right)$ be a sequence of vectors in $\sigma \cap N$, with $v_{1} \in \stackrel{\circ}{\sigma}$, defining the monomial mapping $\Phi^{\underline{v}}$ :

$$
\Phi^{\underline{v}}: \mathbf{C}[[\Lambda]] \longrightarrow \mathbf{C}[[\underline{t}]], \quad X^{\lambda} \longmapsto \underline{t}^{\langle\underline{\nu}, \lambda\rangle}:=t_{1}^{\left\langle v_{1}, \lambda\right\rangle} \cdot \ldots \cdot t_{r}^{\left\langle v_{r}, \lambda\right\rangle} .
$$

Then we have that $\Phi^{\underline{v}}\left(\sum_{\lambda \in \Lambda} X^{\lambda}\right)=P_{\left(Z^{\Lambda}, 0\right)}^{\underline{v}}$.

Proof. This is a direct consequence of the existence of a graded isomorphism between $\operatorname{gr}_{v} R$ and $\mathbf{C}[\Lambda]^{\underline{v}}$ (see Proposition 3.2), since the number $\chi(\mathbf{P} J(\underline{a})$ ) is equal to the dimension of the C-vector space $J(\underline{a})$, which is isomorphic to $H(\underline{a})$. It follows that this dimension is the number of elements in the set $\left\{\lambda \in \Lambda \mid\left\langle v_{j}, \lambda\right\rangle=a_{j}, j=1, \ldots, r\right\}$ (which is finite for every $\underline{a} \in \mathbf{Z}_{\geqslant 0}^{r}$ ).

Remark 7. Lemahieu [25] studied the Poincaré series associated to a toric variety $Z^{\Lambda}$ by a finite sequence of monomial valuations $\underline{\nu}$ centered at $0 \in Z^{\Lambda}$, by using Campillo, Delgado and Gusein-Zade's multi-index filtrations. Theorem 4.1 generalizes [25, Theorem 1] to the case when at least one of the monomial valuations in the sequence $\underline{\nu}$ is centered at $0 \in Z^{\Lambda}$.

REMARK 8. It is well known that the generating series of the semigroup $\Lambda, P=\sum X^{\lambda} \in$ $\mathbf{C}[[\Lambda]]$ is a rational function, that is, it belongs to the ring of fractions of $\mathbf{C}[\Lambda]$ (see [36]). It follows from Theorem 4.1 that the Poincaré series $P_{\left(Z^{\Lambda}, 0\right)}^{\underline{\nu}}$ is also a rational function in $\underline{t}$.

For any $k \geqslant 0$ we denote by

$$
\mathcal{J}_{R}^{k}\left(\nu_{1}\right):=R / I_{\nu_{1}}(k+1)
$$

the space of $k$-jets of functions in $R$, relative to the divisorial valuation $\nu_{1}$ (where $I_{\nu_{1}}(k+1)=$ $\left.\left\{\phi \in R \mid \nu_{1}(\phi) \geqslant k+1\right\}\right)$. This generalizes the classical space of $k$-jets of functions $\mathcal{O}_{\mathbf{C}^{d}, 0} / \mathfrak{m}^{k+1}$. The important fact is that $\nu_{1}$ is centered at the origin, and hence the space of $k$-jets $\mathcal{J}_{R}^{k}\left(\nu_{1}\right)$ is a $\mathbf{C}$-vector space of finite dimension $d_{\nu_{1}}(k)$. We often denote $\mathcal{J}_{R}^{k}\left(\nu_{1}\right)$ and $d_{\nu_{1}}(k)$ by $\mathcal{J}_{R}^{k}$ and $d(k)$, respectively, for short. The symbol $\mathbf{P}^{*} L$ denotes the disjoint union of $\mathbf{P} L$ and a point $*$, for $L$ a finite-dimensional vector space. We have natural maps $\pi_{k}: \mathbf{P} R \rightarrow \mathbf{P}^{*} \mathcal{J}_{R}^{k}\left(\nu_{1}\right)$ and $\pi_{k, k^{\prime}}: \mathbf{P}^{*} \mathcal{J}_{R}^{k}\left(\nu_{1}\right) \rightarrow \mathbf{P}^{*} \mathcal{J}_{R}^{k^{\prime}}\left(\nu_{1}\right)$, for $k \geqslant k^{\prime} \geqslant 0$.

Definition 6. A subset $X \subset \mathbf{P} R$ is cylindrical if $X=\pi_{k}^{-1}(Y)$, for some constructible subset $Y \subset \mathbf{P} \mathcal{J}_{R}^{k} \subset \mathbf{P}^{*} \mathcal{J}_{R}^{k}$. The Euler characteristic of the cylindrical set $X$ is defined as $\chi(X):=\chi(Y)$.

This notion is well defined since the map $\pi_{k, k^{\prime}}$ over $\mathbf{P} \mathcal{J}_{R}^{k^{\prime}} \subset \mathbf{P}^{*} \mathcal{J}_{R}^{k^{\prime}}$ is a locally trivial fibration, with fibers equal to complex affine spaces of dimension $d(k)-d\left(k^{\prime}\right)$.

Definition 7. Let $\Phi: \mathbf{P} R \rightarrow G$ be a function with values in an abelian group $G$. We say that $\Phi$ is cylindrical if, for each $g \in G, g \neq 0$, the set $\Phi^{-1}(g) \subset \mathbf{P} R$ is cylindrical. The integral of the cylindrical function $\Phi$ with respect to the Euler characteristic over the space $\mathbf{P} R$ is 
defined if the sum on the right-hand side of the following formula makes sense in $G$ :

$$
\int_{\mathbf{P} R} \Phi d \chi:=\sum_{g \in \Phi(\mathbf{P} R), g \neq 0} \chi\left(\Phi^{-1}(g)\right) g .
$$

In that case we say that the function $\Phi$ is integrable.

We apply this construction for $G=\mathbf{Z}[[\underline{t}]]$ and $\Phi:=\underline{t}^{\underline{\nu}}$, which is well defined on $\mathbf{P} R$.

THEOREM 4.2. We have

$$
P_{\left(Z^{\Lambda}, 0\right)}^{\nu}=\int_{\mathbf{P} R} t^{\underline{\nu}} d \chi
$$

Proof. Notice that $\Phi(\mathbf{P} R)=\left\{t^{\underline{a}} \mid \underline{a} \in\langle\underline{\nu}, \Lambda\rangle\right\}$. Also notice that if $\underline{a} \in\langle\underline{\nu}, \Lambda\rangle$ then

$$
\Phi^{-1}\left(t^{\underline{a}}\right)=\bigcap_{i} I_{\nu_{i}}\left(a_{i}\right) \backslash \bigcup_{i} I_{\nu_{i}}\left(a_{i}+1\right) .
$$

This set is cylindrical since $\Phi^{-1}\left(t^{\underline{a}}\right)=\pi_{a_{1}}^{-1}(\mathbf{P} H(\underline{a}))$, where $H(\underline{a})$ is seen as a subset of $\mathcal{J}_{R}^{a_{1}}\left(\nu_{1}\right)$. Then it follows that $\chi\left(\Phi^{-1}\left(t^{\underline{a}}\right)\right)=\chi(\mathbf{P} H(\underline{a}))=\chi(\mathbf{P} J(\underline{a}))$, since we have an isomorphism $H(\underline{a}) \rightarrow J(\underline{a})$ by Proposition 3.2. It follows that

$$
\int_{\mathbf{P} R} t^{\underline{\nu}} d \chi=\sum_{\underline{a} \in\langle\underline{\nu}, \Lambda\rangle} \chi(\mathbf{P} J(\underline{a})) t^{\underline{a}}=P_{\left(Z^{\Lambda}, 0\right)}^{\nu} .
$$

Remark 9. Notice that the definition of the cylinders of $\mathbf{P} R$ depends on the choice of a valuation $\nu_{1}$ centered at the origin in the sequence $\underline{\nu}$. A different choice of such valuation provides the same integral $\int_{\mathbf{P} R} t^{\underline{\nu}} d \chi$.

\subsection{Quasi-ordinary hypersurface case}

Let $(S, 0)$ denote an irreducible germ of quasi-ordinary hypersurface defined by a normalized quasi-ordinary polynomial $f$. We use the notation of Section 2 for the normalization map of $(S, 0)$. Let $\underline{v}=\left(v_{1}, \ldots, v_{r}\right)$ be a sequence of vectors in $\sigma \cap N$ with $v_{1} \in \stackrel{\circ}{\sigma}$ defining monomial valuations $\underline{\nu}=\left(\nu_{1}, \ldots, \nu_{r}\right)$ and a multi-graded $\operatorname{ring} \operatorname{gr}_{\underline{v}} \mathcal{O}_{S}=\bigoplus_{\underline{a} \in\left\langle\underline{v}, \Gamma_{\pi}\right\rangle} J(\underline{a})$ (see Proposition 3.3). Since $v_{1} \in \stackrel{\circ}{\sigma}$, we have that formula (4.1) holds (the hypothesis $v_{1} \in \stackrel{\circ}{\sigma}$ is also needed in Proposition 3.3). We can define analogously the extended semigroup $\widetilde{\Gamma}$, and the associated Poincaré series $P_{(S, 0)}^{\nu}$ by formula (4.2). By Proposition 3.3, the graded ring $\operatorname{gr}_{\underline{\nu}} \mathcal{O}_{S}$ is graded isomorphic to $\mathbf{C}\left[\Gamma_{\pi}\right]^{\underline{v}}$ (see (3.1)). It follows that $P_{(S, 0)}^{\underline{\nu}}=P_{\left(Z^{\Gamma}, 0\right)}^{\underline{\nu}}$, for $\Gamma_{\pi}$ the semigroup associated to $(S, 0)$ by the quasi-ordinary projection $\pi$ (see Section 2 ). We deduce from Proposition 2.3 and Theorem 4.1 that the Poincaré series is expressed as follows.

Corollary 4.3. We have

$$
P_{(S, 0)}^{\underline{\nu}}=\Phi^{\underline{v}}\left(P_{\Gamma_{\pi}}\right)=\prod_{j=1}^{d} \frac{1}{1-\underline{t}^{\left\langle\underline{v}, e_{j}\right\rangle}} \prod_{i=1}^{g} \frac{1-\underline{t}^{\left\langle\underline{v}, n_{i} \gamma_{i}\right\rangle}}{1-\underline{t}^{\left.\underline{v}, \gamma_{i}\right\rangle}} \in \mathbf{C}(\underline{t}) .
$$

Remark 10. The Poincaré series $P_{(S, 0)}^{\underline{\nu}}$ depends on the generating series $P_{\Gamma_{\pi}}$ and the sequence of vectors $\underline{v}$. If the sequence of vectors $\underline{v}$ spans a lattice of rank $d$, then the Poincaré series $P_{(S, 0)}^{\underline{\nu}}$ has all coefficients equal to one. In this particular case, one can recover $P_{\Gamma_{\pi}}$ 
from $\underline{v}$ and $P_{(S, 0)}^{\underline{\nu}}$. One important issue in the following sections is to find a sequence $\underline{\nu}$ of monomial valuations, defined intrinsically from the germ $(S, 0)$, in such a way that the sequence of vectors $\underline{v}$ spans a lattice of maximal rank $r \leqslant d$. See Example 1 and Subsection 6.2 for cases with $r<d$.

We prove that the Poincaré series is equal to an integral with respect to the Euler characteristic of the projectivization of the space of the local ring $\mathcal{O}_{S}$. First we define the space of $k$-jets of functions in $R=\mathcal{O}_{S}$ relative to the divisorial valuation $\nu_{1}$ by (4.4). Then we define cylindrical sets in $\mathbf{P} R$ and their Euler characteristic using Definition 6 . We have the following result.

THEOREM 4.4. We have

$$
P_{(S, 0)}^{\underline{\nu}}=\int_{\mathbf{P} \mathcal{O}_{S}} t^{\underline{\nu}} d \chi
$$

Proof. Notice that $\Phi(\mathbf{P} R)=\left\{t^{\underline{a}} \mid \underline{a} \in\left\langle\underline{\nu}, \Gamma_{\pi}\right\rangle\right\}$. If $\underline{a} \in\left\langle\underline{\nu}, \Gamma_{\pi}\right\rangle$ then (4.5) holds. By Remark 2 , for any $\gamma \in \Gamma_{\pi}$ we fix an element $\phi_{\gamma} \in R$ such that $\phi_{\gamma}=X^{\gamma} \epsilon_{\gamma}$, with $\epsilon_{\gamma}$ a unit in the integral closure of $R$. Then the set $\left\{\phi_{\gamma} \mid\langle\underline{v}, \gamma\rangle=\underline{a}\right\}$ has exactly $\operatorname{dim}_{\mathbf{C}} J(\underline{a})$ elements for any $\underline{a}$, by Proposition 3.2. These elements have linearly independent images in the space of $a_{1}$-jets of functions, $\mathcal{J}_{R}^{a_{1}}$, which span a $\mathbf{C}$-vector space $L(\underline{a})$ such that $\Phi^{-1}\left(t^{\underline{a}}\right)=\pi_{a_{1}}^{-1}(\mathbf{P} L(\underline{a}))$. Then it follows that $\Phi^{-1}\left(t^{\underline{a}}\right)$ is a cylinder and $\chi\left(\Phi^{-1}\left(t^{\underline{a}}\right)\right)=\chi(\mathbf{P} L(\underline{a}))=\chi(\mathbf{P} J(\underline{a}))$. We deduce that $t^{\nu}$ is integrable and

$$
\int_{\mathbf{P} R} t^{\underline{\underline{\nu}}} d \chi=\sum_{\underline{a} \in\langle\underline{\nu}, \Lambda\rangle} \chi(\mathbf{P} J(\underline{a})) t^{\underline{a}}=P_{\left(Z_{\pi}^{\Gamma}, 0\right)}^{\nu}
$$

\section{Essential divisors}

In this section we recall the definition and characterization of essential divisors on germs of toric varieties and of quasi-ordinary hypersurface singularities. Using the divisorial valuations associated with essential divisors we define a Poincaré series as an analytic invariant of the germ in Section 6.

We give some definitions in the algebraic case. Let $\psi_{i}: W_{i} \rightarrow V$ be two modifications, with $W_{i}$ normal for $i=1,2$. If $E \subset W_{1}$ is a divisor, then $\psi_{2}^{-1} \circ \psi_{1}$ is defined on a dense open subset $E^{\circ}$ of $E$ and then we say that the closure of $\psi_{2}^{-1} \circ \psi_{1}\left(E^{\circ}\right)$ is the center of the divisor $E$ on $W_{2}$. We identify frequently an exceptional divisor with the associated divisorial valuation. For a variety $V$, a resolution of singularities $\phi: V^{\prime} \rightarrow V$ is a proper modification, with $V^{\prime}$ being smooth and such that $\phi$ is an isomorphism over $V-\operatorname{Sing} V$. If $E$ is an irreducible exceptional divisor of $\phi$ then $\phi(E) \subset \operatorname{Sing} V$, that is, $E$ is an exceptional divisor over the singular locus of $V$. If $\phi(E)$ is contracted to a point $x \in \operatorname{Sing} V$, we say that $E$ is an exceptional divisor over $x$. An exceptional divisor $E$ over the singular locus of $V$ (respectively exceptional divisor over a point $x \in \operatorname{Sing} V$ ) is essential (respectively essential over $x$ ) if for all resolution $\phi: V^{\prime} \rightarrow V$ the center of $E$ on $V^{\prime}$ is an irreducible component of $\phi^{-1}$ (Sing $V$ ) (respectively an irreducible component of $\phi^{-1}(x)$ ).

In the normal toric case the essential divisors admit a combinatorial description explained by Bouvier for essential divisors over zero-dimensional orbits (see [3]) and by Ishii and Kollar in general (see [22]). Let $Z_{\sigma}$ be an affine toric variety defined by a rational strictly convex cone $\sigma \subset N_{\mathbf{R}}$ of maximal dimension. We denote by $o_{\sigma}$ the origin of $Z_{\sigma}$. We suppose that $Z_{\sigma}$ is not smooth. Recall that the singular locus of $Z_{\sigma}$ is the union of orbits corresponding to non-regular faces $\tau$ of $\sigma$. We denote by $\preceq_{\sigma}$ the partial order on $N$ defined by $v \preceq_{\sigma} v^{\prime} \Leftrightarrow v^{\prime} \in v+\sigma$. 
Proposition 5.1 (see $[\mathbf{3}, \mathbf{2 2}]$ ). (i) The essential divisors of the toric variety $Z_{\sigma}$ are the divisors corresponding to the minimal elements with respect to the order $\preceq_{\sigma}$ in the set $\mathcal{D}_{\sigma}:=$ $\bigcup_{\tau \text { non-regular }}^{\tau<\sigma} \stackrel{\circ}{\tau} \cap N$

(ii) The essential divisors over $o_{\sigma}$ are the divisors corresponding to the minimal elements with respect to the order $\preceq_{\sigma}$ in the set $\stackrel{\circ}{\sigma} \cap N$.

It is convenient to give the characterization of essential divisors of an affine toric variety of the form $(V, 0)=\left(Z^{\Lambda}, 0\right)$, for $\Lambda$ a semigroup as in Section 1 . We denote by $n: \bar{V} \rightarrow V$ the normalization map. We have that the essential divisors of $V=Z^{\Lambda}$ over 0 coincide with the essential divisors of its normalization $\bar{V}=Z_{\sigma}$ over the closed orbit. The key point to determine the essential divisors over the singular locus is that the preimage of the singular locus of $V$ is a union of orbits of $Z_{\sigma}$ (the normalization map $n$ being equivariant). Some of these orbits are contained in the singular locus of the normalization $\bar{V}=Z_{\sigma}$, while other orbits consist of non-singular points. If $\tau$ is a regular face of $\sigma$, we denote by $v_{\tau}$ the sum of the primitive vectors of $N$ lying on the edges of $\tau$. The following description is due to Ishii (see [20]).

Proposition 5.2. Let us define $\mathcal{D}^{\prime}:=\left\{v_{\tau} \mid \tau\right.$ regular and $\left.\operatorname{orb}(\tau) \subset n^{-1}(\operatorname{Sing} V)\right\}$. Then the essential divisors of $V$ over its singular locus are the divisors corresponding to the minimal elements with respect to the order $\preceq_{\sigma}$ in the set $\mathcal{D}_{\sigma} \cup \mathcal{D}^{\prime}$. Moreover, the elements in $\mathcal{D}^{\prime}$ are all minimal in $\mathcal{D}_{\sigma} \cup \mathcal{D}^{\prime}$.

The notion of essential divisor extends to the complex analytic or algebroid setting (see [21]). We describe the essential divisors associated to an irreducible germ of quasi-ordinary hypersurface. Ishii proved that the essential divisors of $(S, 0)$ over the origin coincide with the essential divisors of the normalization $(\bar{S}, 0)$ over the origin (see $[\mathbf{2 1}])$, where $(\bar{S}, 0)=\left(Z_{\sigma, N}, o_{\sigma}\right)$ by Proposition 2.2. For the essential divisors over the singular locus, the first author proved the following description, which is based on previous results of Ishii in $[\mathbf{2 0}, \mathbf{2 1}]$. The key point as in the affine toric case is that the preimage by the normalization map of the singular locus of $S$ is the germ of a certain union of orbit closures.

Proposition 5.3 (see [16]). The statement of Proposition 5.2 is true when we replace the toric variety $V$ by the germ $(S, 0)$.

\subsection{The essential matrix}

Using the characterization of the essential valuations of $(S, 0)$, we extract some combinatorial information on the characteristic data associated with the quasi-ordinary polynomial $f$. The content of this section is a technical part needed to prove the main results of the paper in the next section.

We consider the ring extension $\mathbf{C}\{\underline{x}\} \rightarrow \mathbf{C}\left\{\sigma^{\vee} \cap M\right\}$, which maps $x_{i} \mapsto X^{e_{i}}$ and was introduced in Section 2.

REMARK 11. We can identify $N_{0}$ with $\mathbf{Z}^{d}$ by taking coordinates with respect to the dual basis of $\left\{e_{i}\right\}_{i=1}^{d}$. By this identification, the vectors in $\sigma \cap N$ are those vectors $v$ in $\mathbf{Z}_{\geqslant 0}^{d}$ such that $\left\langle v, \gamma_{j}\right\rangle \in \mathbf{Z}$ for $j=1, \ldots, g$. We denote by $u_{i} \in \sigma$ the primitive vector of the lattice $N$ which is orthogonal to $e_{1}, \ldots, \hat{e}_{i}, \ldots, e_{d}$ (where the term $\hat{e}_{i}$ is removed from the list). Notice that the vector $u_{i}$ is an integral multiple of the $i$ th vector of the canonical basis of $\mathbf{Z}^{d}$, for $i=1, \ldots, d$. In particular, if $c<d$ then $u_{c+1}, \ldots, u_{d}$ are vectors in the canonical basis of $\mathbf{Z}^{d}$. 
LEMma 5.4. The preimage of the singular locus by the normalization map is formed by orbit closures of codimension one or two. The orbit closures of codimension one correspond to edges of $\sigma$ of the form $u_{i} \mathbf{R}_{\geqslant 0}$ such that $Z_{i}$ is an irreducible component of the singular locus of $(S, 0)$. The essential divisors over the codimension-one components of $S$ correspond to the lattice vectors $u_{1}, \ldots, u_{s_{1}}$. The orbit closures of codimension two correspond to twodimensional faces of $\sigma$ of the form $\tau_{i, j}:=\operatorname{pos}\left(u_{i}, u_{j}\right)$ such that $Z_{i, j}$ is an irreducible component of the singular locus of $(S, 0)$. There are $n_{g}-1$ essential divisors over each codimension-two component of $\operatorname{Sing} S$, where $n_{g}$ is the last characteristic integer of Definition 2.

(i) If $n_{g}>2$, any two vectors in $\tau_{i, j}^{\circ} \cap N$ defining essential valuations are linearly independent.

(ii) If $n_{g}=2$, there is exactly one essential valuation over any irreducible component of the form $Z_{i, j}$, which is defined by the vector $\left(u_{i}+u_{j}\right) / 2$.

Proof. We use the notation and results on the structure of the singular locus, Theorem 2.1 (see [26, Theorem 7.3 and Corollary 7.3.3]) combined with the description of the essential divisors of Proposition 5.3. We have a natural isomorphism of pairs

$$
\left(\tau_{i, j}, N \cap \tau_{i, j}\right) \longrightarrow\left(\mathbf{R}_{\geqslant 0}^{2},\left\{\left(v_{1}, v_{2}\right) \in \mathbf{Z}_{\geqslant 0}^{2} / v_{1}+v_{2}=0 \bmod n_{g}\right\}\right),
$$

which is deduced from Remark 11 by the following argument: if $v=\left(v_{1}, \ldots, v_{d}\right) \in \tau_{i, j} \cap N$, for $\tau_{i, j}$ a face of $\sigma$ corresponding to a two-dimensional component, then we have that $\left\langle v, \gamma_{k}\right\rangle=0$ for $k=1, \ldots, g-1$ and $\left\langle v, \gamma_{g}\right\rangle=1 / n_{g}\left(v_{i}+v_{j}\right)$, since by Theorems 2.1 and 2.4 the coordinates $i$ and $j$ of $\gamma_{g}$ are equal to $1 / n_{g}$

It follows immediately from this and Proposition 5.3 that there are exactly $n_{g}-1$ minimal vectors in the set $\stackrel{\circ}{\tau}_{i, j} \cap N$ with respect to the order $\preceq_{\sigma}$, and, if $n_{g}>2$, any two of these vectors are linearly independent. If $n_{g}=2$, then there is only one minimal vector $v$ such that $v=(1 / 2)\left(u_{i}+u_{j}\right)$. Notice that in the quasi-ordinary case the cone $\tau_{i, j}$, corresponding to a codimension-two component of the singular locus of $S$, is never regular for the lattice $N$.

DEFINITION 8. We denote by $w_{1}, \ldots, w_{s_{1}}$ (respectively $w_{s_{1}+1}, \ldots, w_{s_{1}+s_{2}}$ ) the sequence of integral vectors in $\sigma \cap N$ corresponding to essential valuations of the germ $(S, 0)$ centered on codimension-one (respectively two) components of Sing $S$. We denote by $w_{s_{1}+s_{2}+1}, \ldots, w_{s_{0}+s_{1}+s_{2}}$ the sequence of integral vectors in $\stackrel{\circ}{\sigma} \cap N$ corresponding to essential valuations of $(S, 0)$ over 0 . We denote by $p$ the number $p=s_{0}+s_{1}+s_{2}$. We denote by $\underline{\omega}$ the sequence of essential valuations

$$
\underline{\omega}:=\left(\omega_{1}, \ldots, \omega_{p}\right),
$$

associated to the sequence of vectors $\underline{w}:=\left(w_{1}, \ldots, w_{p}\right)$.

In the case $d=2$, notice that if the singularity is isolated, $s_{1}=0$, and the notions of essential valuations over the singular locus and over the origin coincide.

By Theorem 2.1 and Lemma 5.4 we have the following remark.

REMARK 12. Notice that $s_{0} \geqslant 1$ by definition of essential valuations of $(S, 0)$ over 0 . The number $s_{1}$ equals the number of codimension-one components of the Sing $S$, while the number $s_{2}$ is greater than or equal to the number $\left(\begin{array}{c}c-s_{1} \\ 2\end{array}\right)$ of codimension-two irreducible components of Sing $S$. We have that $s_{2}=1$ if and only if $c-s_{1}=n_{g}=2$. If $s_{2}=1$ then the following equality holds: $\left\langle w_{s_{1}+1}, e_{c-1}\right\rangle=\left\langle w_{s_{1}+1}, e_{c}\right\rangle=\left\langle w_{s_{1}+1}, \lambda_{g}\right\rangle=1$. 
Definition 9. The matrix $\mathcal{M} \frac{w}{f}:=\left(\left\langle w_{i}, e_{j}\right\rangle\right)_{i=1, \ldots, p}^{j=1, \ldots, d}$ has non-negative integral coefficients and is called the essential matrix associated with the quasi-ordinary polynomial $f$. We denote by $\left\langle\underline{w}, e_{j}\right\rangle$ the vector $\left(\left\langle w_{1}, e_{j}\right\rangle, \ldots,\left\langle w_{p}, e_{j}\right\rangle\right)$.

REMARK 13. The linear map $\psi_{\underline{w}}: M_{\mathbf{Q}} \rightarrow \mathbf{Q}^{p}$, mapping $e_{j} \mapsto\left\langle\underline{w}, e_{j}\right\rangle:=\left(\left\langle w_{1}, e_{j}\right\rangle, \ldots\right.$, $\left.\left\langle w_{p}, e_{j}\right\rangle\right)$, for $j=1, \ldots, d$, has associated matrix $\mathcal{M} \frac{w}{f}$ with respect to the canonical basis.

The following lemma implies that the matrix $\mathcal{M} \frac{w}{f}$ has the block structure shown in Table 1.

Here the first row and column indicate the size of the blocks. The submatrix $D$ is diagonal of maximal rank $s_{1}, B$ is generically of maximal rank $c-s_{1}$, * means non-zero entry and $\underline{1}$ or $\underline{0}$ means all entries are one or zero, respectively. More precisely we have the following result.

Proposition 5.5. If $d>2$ the following statements hold.

(i) The submatrix $D:=\left(\left\langle w_{i}, e_{j}\right\rangle\right)_{1 \leqslant i, j \leqslant s_{1}}$ is diagonal with non-zero determinant.

(ii) The submatrices $\left(\left\langle w_{i}, e_{j}\right\rangle\right)_{s_{1}+1 \leqslant i \leqslant s_{1}+s_{2}}^{1 \leqslant j \leqslant s_{1}}, \quad\left(\left\langle w_{i}, e_{j}\right\rangle\right)_{s_{1}+1 \leqslant i \leqslant s_{1}+s_{2}}^{c<j \leqslant d}$ and $\left(\left\langle w_{i}, e_{j}\right\rangle\right)_{1 \leqslant i \leqslant s_{1}}^{s_{1}<j \leqslant d}$ vanish.

(iii) The submatrix $\left(\left\langle w_{i}, e_{j}\right\rangle\right)_{s_{1}+s_{2}+1 \leqslant i \leqslant p}^{c+1 \leqslant j \leqslant d}$ has all entries equal to one.

(iv) If $c-s_{1} \geqslant 2$ then the submatrix $B:=\left(\left\langle w_{i}, e_{j}\right\rangle\right)_{s_{1}+1 \leqslant i \leqslant s_{1}+s_{2}}^{s_{1}+1 \leqslant j \leqslant c}$ is of rank $c-s_{1}$ if $s_{2} \neq 1$.

(v) The submatrix $\left(\left\langle w_{i}, e_{j}\right\rangle\right)_{1 \leqslant j \leqslant c}^{1 \leqslant j}$ is of rank $c$ if $s_{2} \neq 1$.

Proof. By Lemma 5.4 we have that $w_{1}=u_{1}, \ldots, w_{s_{1}}=u_{s_{1}}$. Then the first assertion follows from this since $\left\langle u_{i}, e_{j}\right\rangle=0 \Leftrightarrow i \neq j$, for $1 \leqslant i, j \leqslant d$.

By Lemma 5.4 we have that, if $s_{1}+1 \leqslant i \leqslant s_{1}+s_{2}$, then there are $s_{1}<j_{1}<j_{2}<c$ such that $w_{i} \in \operatorname{pos}\left(u_{j_{1}}, u_{j_{2}}\right)$. Then assertion (ii) follows from

$$
\left\langle w_{i}, e_{j}\right\rangle=0 \Longleftrightarrow j \neq j_{1}, j_{2} \text { for } 1 \leqslant j \leqslant d .
$$

Notice that if $c<d$ the vectors $u_{c+1}, \ldots, u_{d}$ are part of the canonical vectors in $\mathbf{Z}^{d} \supset N$, by Remark 11. The semigroup $\sigma \cap N$ splits in the form $\left(\sigma^{\prime} \cap N^{\prime}\right) \times \bigoplus_{k=c+1}^{d} \mathbf{Z} u_{k}$, where $\sigma^{\prime}$ (respectively $N^{\prime}$ ) is the intersection of the cone $\sigma$ (respectively the lattice $N$ ) with the subspace spanned by $\left\{u_{1}, \ldots, u_{c}\right\}$. This implies that the vectors $w$ corresponding to essential divisors in $\sigma$ are of the form $w=w^{\prime}+u_{c+1}+\ldots+u_{d}$, where $w^{\prime}$ corresponds to a vector defining an essential divisor in $\sigma^{\prime}$. This shows statement (iii).

For assertion (iv) we have that if $s_{2} \geqslant 2=c-s_{1}$ the vectors $w_{s_{1}+1}, w_{s_{1}+2}$, viewed as forms on $M$, have linearly independent restrictions to the sublattice spanned by $e_{c-1}, e_{c}$.

Otherwise, if $c-s_{1}>2$, then the number $s_{2}$ is greater than or equal to the number of codimension-two components of Sing $S$, which is at least $c-s_{1}$. It is easy to check the result using (5.3).

For statement (v), it is enough to verify the result in the case $c-s_{1}=1$ (and then $s_{2}=0$ ) by (i) and (iv). The forms $w_{1}, \ldots, w_{c-1}, w_{c}$, restricted to the subspace generated by $e_{1}, \ldots, e_{c}$, are

TABLE 1. Block structure of $\mathcal{M} \frac{w}{f}$.

\begin{tabular}{lccc}
\hline & $s_{1}$ & $c-s_{1}$ & $d-c$ \\
\hline$s_{1}$ & $D$ & $\underline{0}$ & $\underline{0}$ \\
$s_{2}$ & $\underline{0}$ & $\underline{*}$ & $\underline{0}$ \\
$s_{0}$ & $\underline{*}$ & $\underline{*}$ & $\underline{1}$ \\
\hline
\end{tabular}


linearly independent by assertion $(\mathrm{i})$, since $w_{c} \in \stackrel{\circ}{\sigma}$ takes non-zero value on $e_{c}$, while $w_{1}, \ldots, w_{c-1}$ vanish on $e_{c}$.

\section{The Poincaré series as an analytical invariant of the singularity}

The definition of Poincaré series of a toric or quasi-ordinary hypersurface singularity depends obviously on the set of vectors $v_{1}, \ldots, v_{r} \in N$. However, we get rid of this dependency by considering the Poincaré series associated with the essential valuations which, in this case, are monomial valuations. In the toric case we have the following definition.

Definition 10. The Poincaré series $P_{\left(Z^{\Lambda}, 0\right)}$ of the germ $\left(Z^{\Lambda}, 0\right)$ is the Poincaré series associated to the sequence of essential valuations of $\left(Z^{\Lambda}, 0\right)$ over the singular locus together with the essential valuations of $\left(Z^{\Lambda}, 0\right)$ over the origin.

This series is well defined since any essential valuation over the origin corresponds to a primitive vector in $\stackrel{\circ}{\sigma} \cap N$, and hence (4.1) holds. Notice that $P_{\left(Z^{\Lambda}, 0\right)} \in \mathbf{Z}\{\underline{t}\}$ is an analytic invariant of the germ $\left(Z^{\Lambda}, 0\right)$ up to permutation of the variables $t_{1}, \ldots, t_{r}$.

If $(S, 0)$ is an analytically irreducible germ of quasi-ordinary hypersurface singularity, we define an intrinsic notion of the Poincaré series.

Definition 11. The Poincaré series $P_{(S, 0)}$ of the germ $(S, 0)$ is the Poincaré series associated to the sequence $(5.2)$ of essential valuations of $(S, 0)$ over the singular locus and the essential valuations of $(S, 0)$ over the origin.

REMARK 14. The indeterminates $\underline{t}=\left(t_{1}, \ldots, t_{p}\right)$ in Definition 11 are distinguished in groups as follows: if $d>2$ then $t_{1}, \ldots, t_{s_{1}}$ (respectively $t_{s_{1}+1}, \ldots, t_{s_{2}}$ ) correspond to the essential valuations in the sequence $\underline{\omega}:=\left(\omega_{1}, \ldots, \omega_{p}\right)$ over codimension-one (respectively two) components of Sing $S$, while $t_{s_{1}+s_{2}+1}, \ldots, t_{p}$ correspond to those essential valuations over the origin in the sequence $\underline{\omega}$. If $d=2$ we consider only two groups as in the case of essential valuations (see Remark 12 ). The Poincaré series of $(S, 0)$ is an analytic invariant of the germ $(S, 0)$, up to permutations of $t_{1}, \ldots, t_{p}$ respecting these groups of indeterminates introduced before.

ExAmple 1. We consider the quadratic cones $y^{2}-x_{1} x_{2}=0$ in $\mathbf{C}^{d+1}$, for $d \geqslant 2$.

(i) If $d=2$ then there is only one essential valuation over the singular locus, which is reduced to the origin. It follows that the associated Poincare series depends on only one variable $t$ and we find that $P_{(S, 0)}=\left(1-t^{2}\right)(1-t)^{-3}$.

(ii) If $d>2$ then there are two essential valuations, one over the singular locus, and the other over the origin, corresponding respectively to the variables $t_{1}, t_{2}$ in the associated Poincaré series $P_{(S, 0)}=\left(1-t_{1}^{2} t_{2}^{2}\right)\left(1-t_{1} t_{2}\right)^{-3}\left(1-t_{2}\right)^{-d+2}$.

The main result of this paper is the following.

Theorem 6.1. The Poincaré series of an irreducible germ $(S, 0)$ of quasi-ordinary hypersurface determines (and is determined by) the sequence of normalized characteristic monomials of $(S, 0)$. 
We analyze the terms appearing in the Poincaré series $P_{(S, 0)}$ when the sum is obtained as in (4.6), from a normalized quasi-ordinary projection and the indeterminates $\underline{t}=\left(t_{1}, \ldots, t_{p}\right)$ are labeled as in Remark 14 .

Lemma 6.2. Suppose that expression (4.6) is deduced from a normalized quasi-ordinary projection $\pi$ of a quasi-ordinary hypersurface of dimension $d>2$ and of equisingular dimension c. We denote by $N_{\pi}$ (respectively by $D_{\pi}$ ) the number of factors of the form $1-\underline{t}^{\underline{a}}$, counted with multiplicities, in the numerator (respectively in the denominator) of expression (4.6). Then we have the following.

(i) There is no cancellation between terms of the form $1-\underline{t}^{\underline{a}}$ in the numerator and denominator of expression (4.6) and $d=D_{\pi}-N_{\pi}$.

(ii) The number $g$ of normalized characteristic exponents is equal to $N_{\pi}$.

(iii) If $s_{2}=0$, then the term $\left(1-t_{s_{1}+s_{2}+1} \cdots t_{p}\right)$ appears in the denominator of (4.6) with multiplicity equal to

$$
\begin{array}{ll}
d-c & \text { if } c=d \text { or }\left\langle\underline{w}, e_{c}\right\rangle \neq\left\langle\underline{w}, e_{d}\right\rangle, \\
d-c+1 & \text { if } c \neq d \text { and }\left\langle\underline{w}, e_{c}\right\rangle=\left\langle\underline{w}, e_{d}\right\rangle .
\end{array}
$$

(iv) If $s_{2}=1$, then the term $\left(1-t_{s_{1}+s_{2}+1} \cdots t_{p}\right)$ appears with multiplicity $d-c$ and the term $\left(1-t_{s_{1}+1} \cdots t_{p}\right)$ appears with multiplicity equal to

$$
\begin{aligned}
& 3 \text { if } g=1 \text { and } \gamma_{1}=(1 / 2,1 / 2,0, \ldots, 0), \\
& 2 \text { otherwise. }
\end{aligned}
$$

(v) If $s_{2} \geqslant 2$ then the term $\left(1-t_{s_{1}+s_{2}+1} \cdots t_{p}\right)$ appears with multiplicity $d-c$.

The other possible factors of the form $1-\underline{t}^{\underline{a}}$ of the numerator or of the denominator appear with multiplicity one.

Proof. By (2.5) we have that $\gamma_{1}<n_{1} \gamma_{1}<\gamma_{2}<n_{2} \gamma_{2}<\ldots<\gamma_{g}<n_{g} \gamma_{g}$ (where $<$ above means $\neq$ and $\leqslant$ coordinate-wise). Since $w_{s_{1}+s_{2}+1} \in \stackrel{\circ}{\sigma}$, we deduce that

$$
\left\langle\underline{w}, \gamma_{1}\right\rangle<\left\langle\underline{w}, n_{1} \gamma_{1}\right\rangle<\ldots<\left\langle\underline{w}, \gamma_{g}\right\rangle<\left\langle\underline{w}, n_{g} \gamma_{g}\right\rangle .
$$

A ray of the form $\mathbf{R}_{\geqslant 0} \gamma_{j}$ may contain only the vector $e_{1}$ in $\left\{e_{1}, \ldots, e_{d}\right\}$ and, if this is the case, since the quasi-ordinary projection is normalized, we have that $e_{1}<\gamma_{1}=\lambda_{1}$ and then

$$
\left\langle\underline{w}, e_{1}\right\rangle<\left\langle\underline{w}, \gamma_{1}\right\rangle \text {. }
$$

A cancellation between terms in the numerator and denominator of (4.6) implies the existence of $j \in\{1, \ldots, g\}$ and $i \in\{1, \ldots, d\}$ such that

$$
\left\langle\underline{w}, n_{j} \gamma_{j}\right\rangle=\left\langle\underline{w}, e_{i}\right\rangle .
$$

By definition, $\gamma_{j}$ is an element in the $\mathbf{Q}$-vector space generated by $e_{1}, \ldots, e_{c}$. Then Proposition 5.5 (iii), implies that any such $i$ has to be at most $c$, since some positive power of $t_{k}$, for $1 \leqslant k \leqslant s_{1}+s_{2}$, divides $\underline{t} \underline{\left.\underline{w}, \gamma_{j}\right\rangle}$. We distinguish two cases as follows.

- If $s_{2} \neq 1$, there is no possible cancellation between terms in the numerator and denominator of (4.6) since the matrix $\left(\left\langle w_{i}, e_{j}\right\rangle\right)_{1 \leqslant i \leqslant p}^{1 \leqslant j \leqslant c}$ is of maximal rank $c$ by Proposition 5.5.

- If $s_{2}=1$ and if $1 \leqslant j<g$ and $1 \leqslant i<c-1$, equality in (6.3) may not occur: the previous discussion implies that $e_{i}=e_{1}$, but then (6.1) and (6.2) give a contradiction. By Remark 12, we have that $n_{g}=2$ and $\underline{t}^{\left\langle\underline{w}, e_{c-1}\right\rangle}=\underline{t}^{\left\langle\underline{w}, e_{c}\right\rangle}=t_{s_{1}+1} \cdot \ldots \cdot t_{p}$. It is clear that this term is not equal to $\underline{t} \underline{\left.\underline{w}, n_{j} \gamma_{j}\right\rangle}$ for $j=1, \ldots, g-1$, while the exponent of $t_{s_{1}+1}$ in $\underline{t} \underline{\left.\underline{w}, 2 \gamma_{g}\right\rangle}$ is equal to two by Remark 12. No cancellation is possible in this case. Example 1 is a particular case of this situation. 
Now assertion (ii) is clear. Statement (iii) follows from Proposition 5.5 (iii), taking into account that if $d>c$ then the column vectors $\left\langle\underline{w}, e_{c}\right\rangle,\left\langle\underline{w}, e_{d}\right\rangle$ of $\mathcal{M} \frac{w}{f}$ may be equal. Statement (v) is similar. The first statement in assertion (iv) follows from Proposition 5.5, (iii) and (iv). The second statement follows from Remark 12 and a simple computation.

Finally, no other factor of the form $1-\underline{t}^{\underline{a}}$ appears with multiplicity greater than one since Proposition $5.5(\mathrm{v})$ implies that the column vectors $\left\langle\underline{w}, e_{j}\right\rangle_{j=1, \ldots, c}$ are linearly independent and determine $\left\langle\underline{w}, \lambda_{i}\right\rangle$ by linearity over $\mathbf{Q}$ (see Remark 13 ).

Proof of Theorem 6.1. After possible simplifications by Lemma 6.2, we have that the Poincaré series of $S$ is expressed uniquely in the form

$$
P_{(S, 0)}=\frac{\left(1-\underline{t}^{\beta_{1}}\right) \cdot \ldots \cdot\left(1-\underline{t}^{\beta_{g}}\right)}{\left(1-\underline{t}^{\alpha_{1}}\right) \cdot \ldots \cdot\left(1-\underline{t}^{\alpha_{d+g}}\right)},
$$

where $d$ is the dimension of $S$ and $g$ is the number of normalized characteristic exponents. By (6.1) we can relabel, if necessary, in order to have $\beta_{1}<\ldots<\beta_{g}$. It may happen that the ray $\beta_{1} \mathbf{R}_{\geqslant 0}$ contains several of the $\alpha_{i}$ or at most one. We denote in the first case by $\alpha_{1}$ the smallest (coordinate-wise) and by $\alpha_{d+1}$ the following one with this property. In the second case we denote by $\alpha_{d+1}$ the unique exponent with this property. By Lemma 6.2 we have that $n_{1} \alpha_{d+1}=\beta_{1}$ for $n_{1}>1$. We repeat the procedure relabeling, if necessary, in order to have that $n_{i} \alpha_{d+i}=\beta_{i}$ for $i=1, \ldots, g$. Notice that the integers $n_{1}, \ldots, n_{g}$ are characteristic integers associated with the normalized characteristic exponents of $(S, 0)$.

We first deal with the case $d>2$. The Poincaré series distinguish the $s_{2}$ indeterminates corresponding to the essential divisors over a codimension-two component of the singular locus of $(S, 0)$ (see Remark 14).

Case 1: $s_{2} \geqslant 2$. By Lemma 6.2, we have that the term $1-t_{s_{1}+s_{2}+1} \cdots t_{p}$ appears in the denominator of $P_{(S, 0)}$ with multiplicity $d-c$. This determines $c$. Notice that by Remark 12 the case $c-s_{1}=n_{g}=2$ is excluded. We relabel then the exponents $\alpha_{1}, \ldots, \alpha_{d}$ in such a way that

$$
\alpha_{d-c+1}=\ldots=\alpha_{d}=(\overbrace{0, \ldots, 0}^{c}, 1, \ldots, 1) .
$$

The matrix $\mathcal{M}$, which has as columns the vectors $\alpha_{1}, \ldots, \alpha_{c}$, is equal to $\left(\left\langle w_{i}, e_{j}\right\rangle\right)_{1 \leqslant i \leqslant p}^{1 \leqslant j \leqslant c}$ up to a permutation of the columns, by Lemma 6.2. By Proposition $5.5(\mathrm{v})$ the matrix $\mathcal{M}$ is of maximal rank $c$. The linear equation $\mathcal{M} \lambda=\alpha_{d+j}$ has exactly one solution, which gives the first $c$ coordinates of $\gamma_{j}$, eventually under a fixed permutation, for $j=1, \ldots, g$. We recover the vectors $\gamma_{1}, \ldots, \gamma_{g}$ in the minimal sequence of generators of the semigroup $\Gamma_{\pi} \subset \mathbf{Q}^{d}$ by adding to each solution $d-c$ zeroes in order to have a $d$-tuple and relabeling the coordinates of the resulting vectors in such a way that (2.2) holds. Formula (2.4) determines from these data the normalized sequence of characteristic exponents.

Case 2: $s_{2}=0$. Then by Lemma 6.2 the term $1-t_{s_{1}+1} \cdots t_{p}$ appears in the denominator of $P_{(S, 0)}$ with multiplicity $d-c^{\prime}$, where $c^{\prime} \in\{c-1, c\}$. We relabel the exponents $\alpha_{1}, \ldots, \alpha_{d}$ in such a way that those corresponding to $t_{s_{1}+1}, \ldots, t_{p}$ appear with the highest index (as in (6.4) above). By Lemma 6.2 the matrix $\mathcal{M}$, with columns the vectors $\alpha_{1}, \ldots, \alpha_{c^{\prime}}$, is equal to $\left(\left\langle w_{i}, e_{j}\right\rangle\right)_{1 \leqslant i \leqslant p}^{1 \leqslant j \leqslant c^{\prime}}$, up to a permutation of the columns. By Proposition $5.5(\mathrm{v})$ the matrix $\mathcal{M}$ is of maximal rank $c^{\prime}$.

In order to determine $c$ we study the linear equation $\mathcal{M} \lambda=\alpha_{d+g}$. If this equation has no solution then $c=c^{\prime}+1$, and then we replace $\mathcal{M}$ by the matrix, denoted with the same letter, with columns the vectors $\alpha_{1}, \ldots, \alpha_{c^{\prime}+1}$. In this case this matrix is equal to $\left(\left\langle w_{i}, e_{j}\right\rangle\right)_{1 \leqslant i \leqslant p}^{1 \leqslant j \leqslant c^{\prime}+1}$, 
up to a permutation of the columns, and it is of rank $c^{\prime}+1=c$, by Proposition 5.5 (v). Then in both cases the linear system $\mathcal{M} \lambda=\alpha_{d+j}$ has exactly one solution for $j=1, \ldots, g$ from which we recover the normalized characteristic exponents as in the previous case.

Case 3: $s_{2}=1$. Then the term $1-t_{s_{1}+2} \ldots t_{p}$ appears in the denominator of $P_{(S, 0)}$ with multiplicity $d-c$ and the term $\left(1-t_{s_{1}+1} \ldots t_{p}\right)$ appears also with multiplicity two or three. We relabel the exponents $\alpha_{1}, \ldots, \alpha_{d}$ in such a way that those corresponding to the monomial $t_{s_{1}+2} \ldots t_{p}$ appear with the highest index (as in (6.4) above) followed by those exponents corresponding to the monomial $t_{s_{1}+1} \ldots t_{p}$. Notice that we can distinguish these monomials in the Poincaré series by Remark 14 . We denote by $\xi^{\prime}$ the $s_{1}$-tuple obtained from $\xi \in \mathbf{Q}^{p}$ by keeping only the first $s_{1}$ coordinates. By Lemma 6.2 the matrix $\mathcal{M}$, with columns the vectors $\alpha_{1}^{\prime}, \ldots, \alpha_{s_{1}}^{\prime}$, is equal to $\left(\left\langle w_{i}, e_{j}\right\rangle\right)_{1 \leqslant i \leqslant s_{1}}^{1 \leqslant j \leqslant s_{1}}$, up to a permutation of the columns. By Proposition 5.5 (i) we have that $\mathcal{M}$ is then of maximal rank $s_{1}$. The linear equation $\mathcal{M} \lambda=\alpha_{d+j}^{\prime}$ has exactly one solution, for $j=1, \ldots, g-1$, which gives the first $c-2=s_{1}$ coordinates of $\gamma_{j}^{\prime}$, eventually under a fixed permutation, for $j=1, \ldots, g$. We form $d$-tuples from these terms putting zeroes in the new coordinates for $j=1, \ldots, g-1$. For $j=g$ we put two new coordinates equal to $1 / 2$ and the remaining ones equal to zero (we use Remark 12, Theorem 2.1 and equation (2.4)). Then the proof is complete as in the case $s_{2} \geqslant 2$.

Finally we deal with the surface case. If the singularity is normal, then it is isolated and hence it follows that $s_{1}=0, c=2$ and the essential valuations over the singular locus coincide with the essential valuations over the origin. In this case the singularity is isomorphic to $y^{n}-x_{1} x_{2}$ by [26]. It is an $A_{n-1}$ singularity possessing $n-1$ irreducible exceptional divisors in the minimal resolution, all of them essential divisors corresponding to integral lattice vectors pairwise linearly independent if $n>2$ (see [12]). If $n=2$ then there is only one such irreducible exceptional divisor in the minimal resolution and hence only one essential valuation (see Example 1). If the singularity is not normal, then its singular locus of $S$ has one or two irreducible components. If we have two components then the assertion is a consequence of Lemma 5.4: the corresponding vectors $w_{1}=u_{1}, w_{2}=u_{2}$ are obviously linearly independent. Otherwise, if there is only one component, corresponding to the vector $w_{1}=u_{1}$, then we have that the vector $w_{p} \in \stackrel{\circ}{\sigma}$ is linearly independent of $w_{1}$. In any of these cases, with the exception of the quadratic cone $y^{2}=x_{1} x_{2}$, the matrix $\mathcal{M}$ with columns the vectors $\alpha_{1}, \ldots, \alpha_{p}$ is equal to a permutation of the columns of $\left(\left\langle w_{i}, e_{j}\right\rangle\right)_{1 \leqslant i \leqslant p}^{1 \leqslant j \leqslant 2}$. Since this matrix is non-singular, we recover the vectors $\gamma_{1}, \ldots, \gamma_{g}$, up to a fixed permutation of the coordinates by resolving the linear systems $\mathcal{M} \gamma=\alpha_{2+i}$ for $i=1, \ldots, g$. Then we conclude as in the previous cases.

Notice that the procedure is completely algorithmic, once the reduced form of the Poincaré series is given, and uses only elementary linear algebra operations.

Let $(S, 0) \subset\left(\mathbf{C}^{d+1}, 0\right)$ be an irreducible germ of a quasi-ordinary hypersurface singularity defined by a normalized quasi-ordinary polynomial $f \in \mathbf{C}\left\{x_{1}, \ldots, x_{d}\right\}[y]$. We denote by $\left(S^{\prime}, 0\right)$ the $d^{\prime}$-dimensional germ obtained from $S$ by intersecting with $x_{d-d^{\prime}+1}=\ldots=x_{d}=0$. The following corollary is then immediate.

COROLlary 6.3. The germ $(S, 0)$ is a topologically equisingular deformation of a quasiordinary hypersurface singularity $\left(S^{\prime}, 0\right)$ of dimension $1 \leqslant d^{\prime} \leqslant d$ if and only if

$$
P_{(S, 0)}\left(t_{1}, \ldots, t_{p}\right)=\left(\frac{1}{1-t_{s_{1}+s_{2}+1} \ldots t_{p}}\right)^{d-d^{\prime}} P_{\left(S^{\prime}, 0\right)}\left(t_{1}, \ldots, t_{p}\right),
$$

where the indeterminates $t_{1}, \ldots, t_{p}$ are distributed in groups (see Remark 14) in the same form for $(S, 0)$ and $\left(S^{\prime}, 0\right)$. 
Notice that the minimal possible value of $d^{\prime}$ in Corollary 6.3 is equal to the equisingular dimension of $(S, 0)$ (see Section 2).

\subsection{The Poincaré series and the zeta function}

In this section we compare the zeta function of geometric monodromy of an irreducible quasiordinary polynomial function $f:\left(\mathbf{C}^{d+1}, 0\right) \rightarrow(\mathbf{C}, 0)$ with the Poincaré series of the germ $f=0$ at 0 , for $d \geqslant 2$. This zeta function is studied by McEwan and Némethi in [29]. See also [18] for a description when $f$ is not irreducible.

We suppose that $f \in \mathbf{C}\left\{x_{1}, \ldots, x_{d}\right\}[y]$ is a normalized irreducible quasi-ordinary polynomial, with characteristic exponents $\lambda_{j}=\left(\lambda_{j, 1}, \ldots, \lambda_{j, d}\right) \in \mathbf{Q}^{d}$ for $j=1, \ldots, g$.

Theorem 6.4 (see $[\mathbf{2 9}]$, Theorem A). The zeta function of geometric monodromy of $f$ : $\left(\mathbf{C}^{d+1}, 0\right) \rightarrow(\mathbf{C}, 0)$ is equal to

$$
\zeta(f)(t)=\zeta\left(\left.f\right|_{x_{2}=\cdots=x_{d}=0}\right)(t) .
$$

More precisely, there are two cases:

(A) If $\lambda_{1,2} \neq 0$ then we have that $\zeta(f)(t)=1-t^{n}$ with $n=\operatorname{deg}_{y}(f)$.

(B) If $\lambda_{1,2}=0$, we denote by $i_{0}$ the maximum among those indices $1 \leqslant i \leqslant g$ such that $\lambda_{i, 2}=0$ and by $h\left(x_{1}, y\right) \in \mathbf{C}\left\{x_{1}\right\}[y]$ the irreducible Weierstrass polynomial, with the Puiseux expansion $y=\sum_{\lambda_{i} \leqslant \lambda_{i_{0}}} x_{1}^{\lambda_{i, 1}}$. Then we have that $\zeta(f)(t)=\zeta(h)\left(t^{n / n_{1} \cdot \ldots \cdot n_{i_{0}}}\right)$.

Denote by $P$ the result of substituting the indeterminates $t_{1}, \ldots, t_{p}$ by $t$ in the Poincaré series $P_{(S, 0)}$. Remark that $P$ coincides with the Poincaré series $P_{(S, 0)}^{w}$, where $w:=w_{1}+\ldots+w_{p}$ is the sum of all vectors in $\sigma \cap N$ corresponding to essential valuations. Note that $w \in \stackrel{\circ}{\sigma} \cap N$. Let us write $w=\left(b_{1}, \ldots, b_{d}\right) \in \mathbf{Z}^{d}$, for $b_{i}=\sum_{j=1}^{r} w_{i, j}$, where the coordinates $w_{i}=\left(w_{i, 1}, \ldots, w_{i, d}\right)$ are defined by Remark 11 . Then $P$ is expressed as follows:

$$
P=\left(\frac{\prod_{j=1}^{i_{0}}\left(1-t^{b_{1} n_{j} \gamma_{j, 1}}\right)}{\prod_{j=1}^{i_{0}}\left(1-t^{b_{1} \gamma_{j, 1}}\right)\left(1-t^{b_{1}}\right)}\right) \prod_{j=i_{0}+1}^{g}\left(\frac{1-t^{\left\langle w, n_{j} \gamma_{j}\right\rangle}}{1-t^{\left\langle w, \gamma_{j}\right\rangle}}\right) \prod_{j=2}^{d}\left(\frac{1}{1-t^{b_{j}}}\right) .
$$

Corollary 6.5. The function $\zeta(f)\left(t^{b_{1} / n}\right)$ appears as a factor in the expansion (6.5) of $P$ in the following way:

(A)

$$
\lambda_{1,2} \neq 0, \quad P=\frac{1}{\zeta(f)\left(t^{b_{1} / n}\right)}\left(\frac{\prod_{j=1}^{i_{0}}\left(1-t^{b_{1} n_{j} \gamma_{j, 1}}\right)}{\prod_{j=1}^{i_{0}}\left(1-t^{b_{1} \gamma_{j, 1}}\right)}\right) \prod_{j=i_{0}+1}^{g}\left(\frac{1-t^{\left\langle w, n_{j} \gamma_{j}\right\rangle}}{1-t^{\left\langle w, \gamma_{j}\right\rangle}}\right) \prod_{j=2}^{d}\left(\frac{1}{1-t^{b_{j}}}\right) .
$$

(B)

$$
\lambda_{1,2}=0, \quad P=\zeta(h)\left(t^{b_{1} / \operatorname{deg}(h)}\right) \prod_{j=i_{0}+1}^{g}\left(\frac{1-t^{\left\langle w, n_{j} \gamma_{j}\right\rangle}}{1-t^{\left\langle w, \gamma_{j}\right\rangle}}\right) \prod_{j=2}^{d}\left(\frac{1}{1-t^{b_{j}}}\right) .
$$

Notice that the Poincare series $P$ encodes the information provided by the zeta function exactly when $i_{0}=g$, that is, in the case of an equisingular family of plane branches, that is, $c=1$. 
Question. Is it possible to find a notion of zeta function which is more adapted to nonisolated singularities and which in the case of quasi-ordinary hypersurface singularities encodes the embedded topological type of information recovered by the Poincaré series?

\subsection{Example}

Let us consider a three-dimensional germ $(S, 0) \subset\left(\mathbb{C}^{4}, 0\right)$, which in coordinates $\left(x_{1}, x_{2}, x_{3}, y\right)$ has equation $f=0$, where $f=\left(y-x_{1}\right)^{3}-x_{1}^{11} x_{2}\left(1+x_{3}\right)$. The characteristic exponents corresponding to the quasi-ordinary projection $\left(x_{1}, x_{2}, x_{3}, y\right) \mapsto\left(x_{1}, x_{2}, x_{3}\right)$ are $\lambda_{1}=\left(\frac{1}{3}, 0,0\right)$ and $\lambda_{2}=\left(\frac{5}{9}, \frac{1}{9}, 0\right)$. The generators of the semigroup $\Gamma_{\pi}$ are $\gamma_{1}=\left(\frac{1}{3}, 0,0\right)$ and $\gamma_{2}=\left(\frac{11}{9}, \frac{1}{9}, 0\right)$ and the characteristic integers are $n_{1}=3$ and $n_{2}=9$. By Theorem 2.1, the singular locus of $(S, 0)$ has only one component, which is of codimension one, defined by the intersection with $x_{1}=0$.

We identify the lattice $N_{0}$ defined in Section 2 with $\mathbf{Z}^{3}$ using Remark 11 . Then the lattice $\sigma \cap N$ is a subsemigroup of $\mathbf{Z}_{\geqslant 0}^{3}$ and $N$ is viewed as a sublattice of $\mathbf{Z}^{3}$. We find that the vectors $v_{1}=(9,0,0), v_{2}=(3,3,0)$ and $v_{3}=(0,0,1)$ are a basis of $N$ (for this purpose we can use the algorithms introduced by Popescu-Pampu in [35]). By Lemma 5.4, the essential divisor over the codimension-one component of $S$ corresponds to the vector $v_{1}$, and the essential divisors over the origin correspond to the minimal vectors in $\stackrel{\circ}{\sigma} \cap N$ with respect to the order $\preceq_{\sigma}$. We find in this case only one minimal element $w=(3,3,1)$.

The essential matrix associated with $\bar{w}:=(v, w)$ is

$$
\mathcal{M}_{f}^{\bar{w}}=\left(\begin{array}{lll}
9 & 0 & 0 \\
3 & 3 & 1
\end{array}\right)
$$

and the Poincaré series is

$$
P_{(S, 0)}=\frac{\left(1-t_{1}^{9} t_{2}^{3}\right)\left(1-t_{1}^{99} t_{2}^{36}\right)}{\left(1-t_{1}^{9} t_{2}^{3}\right)\left(1-t_{2}^{3}\right)\left(1-t_{2}\right)\left(1-t_{1}^{3} t_{2}\right)\left(1-t_{1}^{11} t_{2}^{4}\right)}
$$

Notice that the factor $\left(1-t_{1}^{9} t_{2}^{3}\right)$ appears in both the numerator and denominator of (6.6). This does not contradict Lemma 6.2 since the quasi-ordinary projection is not normalized (see Section 2). By simplifying this factor we obtain that

$$
P_{(S, 0)}=\frac{1-t_{1}^{99} t_{2}^{36}}{\left(1-t_{2}\right)\left(1-t_{2}^{3}\right)\left(1-t_{1}^{3} t_{2} t_{1}^{3}\right)\left(1-t_{1}^{11} t_{2}^{4}\right)} .
$$

We now apply the proof of Theorem 6.1 as an algorithm starting from expression (6.7). We deduce that $d=3$ and $g=1$. Moreover, since we have only one relation of the form $\left(t_{1}^{11} t_{2}^{4}\right)^{9}=$ $t_{1}^{99} t_{2}^{36}$, we have that the first normalized characteristic integer is 9 . By hypothesis we know that $s_{2}=0$. The term $1-t_{2}$ appears in the denominator with multiplicity one. The matrix $\mathcal{M}$ is equal to

$$
\mathcal{M}=\left(\begin{array}{ll}
3 & 0 \\
9 & 3
\end{array}\right)
$$

The linear system $\mathcal{M} \lambda=(11,4)^{t}$ has a unique solution $\left(\frac{11}{3}, \frac{1}{9}\right)$, and therefore the normalized sequence of characteristic exponents associated to $(S, 0)$ in this case is reduced to $\lambda_{1}^{\prime}=$ $\left(\frac{11}{3}, \frac{1}{9}, 0\right)$, the normalized characteristic sequence associated to $(S, 0)$.

Acknowledgements. The authors are grateful to A. Campillo, F. Delgado, S. M. GuseinZade, A. Lemahieu, P. Popescu-Pampu and B. Teissier for helpful suggestions and discussions. The second author thanks the Universidad Complutense de Madrid and its research team Grupo Singular for their hospitality. 


\section{References}

1. S. S. Abhyankar, 'On the ramification of algebraic functions', Amer. J. Math. 77 (1995) 575-592.

2. C. BAn,, 'A Whitney stratification and equisingular family of quasi-ordinary singularities', Proc. Amer. Math. Soc. 117 (1993) 305-311.

3. C. Bouvier, 'Diviseurs essentiels, composantes essentielles des variétés toriques singulères', Duke Math. J. 91 (1998) 609-620.

4. C. Bouvier and G. Gonzalez-Sprinberg, 'Système générateur minimal, diviseurs essentiels et G-désingularisations de variétés toriques', Tôhoku Math. J. 47 (1995) 125-149.

5. A. Campillo, F. Delgado and S. M. Gusein-Zade, 'The Alexander polynomial of a plane curve singularity via the ring of functions on it', Duke Math. J. 117 (2003) 125-156.

6. A. Campillo, F. Delgado and S. M. Gusein-Zade, 'Poincaré series of a rational surface singularity', Invent. Math. 155 (2004) 41-53.

7. A. Campillo, F. Delgado and S. M. Gusein-Zade, 'Poincaré series of curves on rational surface singularities', Comment. Math. Helv. 80 (2005) 95-102.

8. S. D. Cutkosky, J. Herzog and A. Reguera, 'Poincaré series of resolutions of surface singularities', Trans. Amer. Math. Soc. 356 (2004) 1833-1874.

9. W. Ebeling and S. M. Gusein-Zade, 'Poincaré series and zeta function of the monodromy of a quasihomogeneous singularity', Math. Res. Lett. 9 (2002) 509-513.

10. W. Ebeling and S. M. Gusein-Zade, 'Monodromies and Poincaré series of quasihomogeneous complete intersections', Abh. Math. Sem. Univ. Hamburg 74 (2004) 175-179.

11. G. Ewald, Combinatorial convexity and algebraic geometry (Springer, Berlin, 1996).

12. W. Fulton, Introduction to toric varieties, Annals of Mathematical Studies 131 (Princeton University Press, Princeton, 1993).

13. Y.-N. GaU, 'Embedded topological clasification of quasi-ordinary singularities', Mem. Amer. Math. Soc. 388 (1988) 109-129.

14. P. D. González Pérez, 'The semigroup of a quasi-ordinary hypersurface', J. Inst. Math. Jussieu 2 (2003) 383-399.

15. P. D. González Pérez, 'Toric embedded resolutions of quasi-ordinary hypersurface singularities', Ann. Inst. Fourier (Grenoble) 53 (2003) 1819-1881.

16. P. D. González PÉrez, 'Bijectiveness of the Nash map for quasi-ordinary hypersurface singularities', Int. Math. Res. Not. 19 (2007) Article ID: rnm076-13.

17. P. D. González Pérez and G. Gonzalez-Sprinberg, 'Analytical invariants of quasi-ordinary hypersurface singularities associated to divisorial valuations', Kodai Math. J. 27 (2004) 164-173.

18. P. D. GonzÁlez Pérez, L. J. McEwan and A. Némethi, 'The zeta-function of a quasi-ordinary singularity. II', Topics in algebraic and noncommutative geometry, Luminy/Annapolis, MD, 2001, Contemporary Mathematics 324 (American Mathematical Society, Providence, RI, 2003) 109-122.

19. S. M. Gusein-Zade, F. Delgado and A. Campillo, 'On the monodromy of a plane curve singularity and the Poincaré series of its ring of functions', Funct. Anal. Appl. 33 (1999) 56-57.

20. S. Ishiı, 'Arcs, valuations and the Nash map', J. Reine Angew. Math. 588 (2005) 71-92.

21. S. IsHiI, 'The local Nash problem on arc families of singularities', Ann. Inst. Fourier (Grenoble) 56 (2006) $1207-1224$

22. S. Ishi and J. Kollar, 'The Nash problem on arc families of singularities', Duke Math. J. 120 (2003) 601-620.

23. H. W. E. JunG, 'Darstellung der Funktionen eines algebraischen Körpers zweier unabhaängigen Veränderlichen $x, y$ in der Umgebung einer stelle $x=a, y=b^{\prime}$, J. Reine Angew. Math. 133 (1908) 289-314

24. K. KiyeK and M. Micus, Semigroup of a quasiordinary singularity, Topics in Algebra, Banach Center Publications 26(2) (PWN-Polish Scientific Publishers, Warsaw, 1990).

25. A. Lemahieu, 'The Poincaré series of a toric variety', J. Algebra 315 (2007) 683-697.

26. J. Lipman, 'Topological invariants of quasi-ordinary singularities', Mem. Amer. Math. Soc. 74 (1988) 1-107.

27. J. Lipman, 'Equisingularity and simultaneous resolution of singularities', Resolution of singularities, Obergurgl, 1997, Progressions in Mathematics 181 (Birkhäuser, Basel, 2000) 485-505.

28. L. J. McEwAN and A. NÉMEThi, 'Some conjectures about quasi-ordinary singularities', Topics in algebraic and noncommutative geometry, Luminy/Annapolis, MD, 2001, Contemporary Mathematics 324 (American Mathematical Society, Providence, RI, 2003) 185-193.

29. L. J. MCEWAn and A. NÉmethi, 'The zeta function of a quasi-ordinary singularity', Compos. Math. 140 (2004) 667-682.

30. J. F. NAsh JR, 'Arc structure of singularities', A celebration of John F. Nash, Jr., Duke Math. J. 81 (1995) $31-38$

31. A. NÉmethi, 'Poincaré series associated with surface singularities', Singularities I: algebraic and analytic aspects. International Conference in Honor of the 60th Birthday of Lê Dùng Tráng, January 8-26, 2007, Cuernavaca, Mexico (eds J.-P. Brasselet, J.L. Cisneros-Molina, J. Seade, B. Teissier), Contemporary Mathematics 474 (American Mathematical Society, Providence, RI, 2008) 271-298

32. T. ODA, Convex bodies and algebraic geometry, Annals of Mathematical Studies 131 (Springer, Berlin, 1988).

33. P. POPESCU-PAMPU, 'On the invariance of the semigroup of a quasi-ordinary surface singularity', C.R Acad. Sci. Paris, Ser. I 334 (2002) 1101-1106. 
34. P. Popescu-PAmpu, 'On the analytical invariance of the semigroups of a quasi-ordinary hypersurface singularity', Duke Math. J. 124 (2004) 67-104.

35. P. Popescu-PAmpu, 'On higher dimensional Hirzebruch-Jung singularities', Rev. Mat. Complut. 18 (2005) 209-232.

36. R. P. Stanley, Combinatorics and commutative algebra, 2nd edn, Progress in Mathematics 41 (Birkhäuser Boston, Boston, MA, 1996).

37. B. Teissier, 'The monomial curve and its deformations', Appendix, Le Problme des modules pour les branches planes (Hermann, Paris, 1986).

P. D. González Pérez

Instituto de Ciencias

Matemáticas-CSIC-UAM-UC3M-UCM

Departamento de Algebra

Facultad de Ciencias Matemáticas

Universidad Complutense de Madrid

Plaza de las Ciencias 3

28040 Madrid

Spain

pgonzalez@mat.ucm.es

\section{F. Hernando}

School of Mathematical Sciences

Aras Na Laoi

University of Cork

Ireland

f.hernando@ucc.ie 